\title{
ASIMETRÍAS EN EL TRASPASO DE PRECIOS DE MATERIAS PRIMAS EN COSTA RICA, ¿INFLUYE EL NIVEL DE COMPETENCIA?
}

\author{
Cristian Álvarez Corrales ${ }^{1}$ \\ Manfred Esquivel Monge ${ }^{2}$
}

\begin{abstract}
RESUMEN
Se estudia la presencia de asimetrías en el traspaso de precios de materias primas a precios de bienes de consumo en Costa Rica, mediante modelos multiecuacionales estructurales de rezagos distribuidos. Esto en contraposición a la literatura tradicional que emplea modelos VAR censurados, pues estos últimos generan estimaciones del traspaso asintóticamente sesgadas. La evidencia sugiere que el traspaso al índice de precio al consumidor no muestra evidencia de asimetría, pero en productos asociados a trigo, maíz, arroz y fertilizantes el traspaso es asimétrico; además, se encuentra que la asimetría se acentúa en los mercados donde existe alta concentración de oferentes.
\end{abstract}

PALABRAS CLAVE: MATERIAS PRIMAS, TRASPASO DE PRECIOS, ASIMETRÍAS, ORGANIZACIÓN INDUSTRIAL, MODELOS ESTRUCTURALES.

CLASIFICACIÓN JEL: E31, C31, L11

\section{ABSTRACT}

We study the presence of asymmetries in the pass-through of commodities prices to domestic prices in Costa Rica by means of structural distributed lags models to deal with the asymptotic bias that emerges when censored VAR models (the traditional approach) are employed to capture asymmetries. The pass-through to consumer price index does not show evidence of asymmetry, but in products associated with wheat, maize, rice and fertilizers the pass-through is asymmetric. We also found that the asymmetry becomes higher as the concentration of suppliers increases.

KEYWORDS: COMMODITIES, PASS-THROUGH OF PRICES, ASYMMETRIES, INDUSTRIAL ORGANIZATION, STRUCTURAL MODELS.

JEL CLASIFICATION: E12, E31, E52

1 Banco Central de Costa Rica, Departamento de Investigación Económica, División Económica; Código Postal 10058-1000; Costa Rica. alvarezcc@bccr.fi.cr

2 Banco Central de Costa Rica, Departamento de Investigación Económica, División Económica; Código Postal 10058-1000; Costa Rica. esquivelmm@bccr.fi.cr 


\section{INTRODUCCIÓN}

Las variaciones de precio de materias primas (MP) pueden contener información sobre la dinámica futura de precios nacionales debido a varias razones. En primer término, los precios de las MP son parte de los costos de producción de las empresas, así que los choques sobre estas deberían impactar vía la cadena de producción. En segundo lugar, los precios nacionales tienden a mostrar rigideces ocasionadas por las características de los contratos laborales y efectos de costos de menú, mientras que los mercados de MP por lo general son más flexibles. De ahí que sea esperable que estos últimos reflejen de manera adelantada cambios en las condiciones de oferta $y$ demanda que luego se transmiten a los indicadores de inflación. Cutler, Chan y Li (2005) señalan que algunas MP como el oro se usan como cobertura contra choques inflacionarios. Así que incrementos en expectativas de inflación se asocian con repuntes en el precio de MP y señalarían presión inflacionaria de forma adelantada, al menos en países con mercados financieros desarrollados.

Según Cutler et al. (2005) la porción de los choques sobre precios de MP que termina trasmitiéndose a precios finales depende, entre otros factores, de la expectativa de los agentes económicos respecto a la respuesta de la autoridad monetaria a esos choques. Una reacción fuerte y rápida de la autoridad monetaria se asociaría con un traspaso reducido. Sobre la magnitud del traspaso también influyen: el momento del ciclo económico, si el país es productor de esa MP, la relación entre el precio en la MP y el tipo de cambio (TC), entre otros.

La literatura empírica documenta, para la economía estadounidense, un declive en el traspaso de precios de MP a partir de mitad de los años ochenta (Blomberg \& Harris, 1995; Furlong \& Ingenito, 1996). Esta baja se atribuye a una reducción en la intensidad con que esa economía utiliza MP, a cambios en la forma en que la Fed responde a tales choques y a un uso menos intensivo de las MP como cobertura contra inflación debido al desarrollo de instrumentos financieros más aptos para tal fin.

En contraste, en economías en desarrollo se documenta un fortalecimiento histórico del traspaso. Cutler et al. (2005) así lo evidencian para China Continental y Hong Kong. Los autores atribuyen tal fortalecimiento al grado de apertura de esas economías, al mayor peso relativo de los alimentos en el índice de precios al consumidor (IPC) y al mayor tamaño relativo de industrias manufactureras intensivas en el uso de MP.

En Costa Rica existen razones para esperar un traspaso reducido y con asimetría de signo. En primer término, la economía costarricense es pequeña y tomadora de precios en mercados internacionales de MP, así que incrementos en expectativas de inflación y un supuesto refugio en MP de parte de agentes costarricense no tendría efecto alguno sobre su precio. En segundo lugar, el peso del grupo "bebidas y alimentos" en el IPC de Costa Rica es menos de la mitad del que tiene en China Continental, donde su importancia relativa es casi el 50\%. Por otra parte, la importancia de la manufactura en la economía costarricense es inferior a la de China Continental. Además, en comparación con la de China, la matriz energética costarricense es poco dependiente de hidrocarburos. Así que los choques sobre precios de energía y metales no deberían impactar tanto los costos de producción ni los precios finales. Finalmente, debido a que algunas industrias de la economía costarricense que son intensivas en el uso de MP tienen un esquema de organización industrial poco competitivo,es esperable que sus precios reflejen con mayor facilidad los choques positivos que los negativos sobre MP.

Conocer la magnitud del traspaso de precios de MP a nivel microeconómico (de producto o industria) resulta relevante pues permitiría identificar aquellas industrias en las que la estructura de organización industrial podría estar provocando asimetrías de signo. En caso de que tales asimetrías fuesen provocadas por estructuras monopolísticas, el mecanismo de precios de mercado estaría conduciendo resultados sub óptimos en la asignación de recursos. Tal situación ameritaría considerar políticas que subsanen esas imperfecciones. 
La posible asimetría en el impacto de las variaciones de precio de MP sobre variables domésticas ha sido ampliamente estudiada. Si bien los trabajos evaluaron el impacto sobre variables reales, los avances logrados en las metodologías empleadas pueden extenderse al estudio del efecto sobre otras variables.

Uno de los primeros trabajos que, utilizando datos que incluían las reducciones de precio del petróleo de 1980-8633, analiza el tema de la asimetría en el traspaso de precios de MP es Mork (1989). El autor emplea modelos VAR que censuran las variaciones negativas de precio del petróleo, y concluye que el impacto sobre el crecimiento del PIB no necesariamente es simétrico en el signo de los choques. En relación a estimaciones que utilizaban modelos VAR estándar, los resultados de Mork (1989) se apegaban más a los datos observados. Desde entonces, los modelos VAR censurados fueron ampliamente utilizados para analizar el impacto de variaciones en precios de MP sobre variables domésticas (ver por ejemplo Bernanke, et al. 1997).

Varios autores han propuesto modificaciones a la propuesta original de Mork (1989). Por ejemplo, Lee, Ni y Rati (1995) proponen una forma de modelar asimetrías en volatilidad en la que los choques se generan reescalando las series originales de precio por un indicador de volatilidad obtenido a partir de un modelo GARCH. La intuición es que las variaciones de precio de MP tendrán menor efecto si su variancia condicional es grande pues ante tal coyuntura esos cambios serán percibidos como temporales. Los autores concluyen que los choques no esperados tienen un mayor impacto en tanto más estable hayan sido los precios recientemente. Además, hallan que solo choques positivos (reescalados) tienen efecto estadísticamente significativo.

Otra modificación a la propuesta de Mork (1989) es la de Hamilton (1996, 2003). Según el autor, son los incrementos netos del precio del petróleo los que afectan el producto real. La serie de precio que utiliza en sus estimaciones es :

$$
\bar{\pi}_{t}^{e}=\max (0, P)
$$

Donde

$$
P=\log \left(\pi_{t}^{e}\right)-\max \left[\log \left(\pi_{t-1}^{e}\right), \log \left(\pi_{t-2}^{e}\right), \ldots \log \left(\pi_{t-12}^{e}\right)\right]
$$

$y \pi_{t}^{e}$ corresponde al nivel del índice de precios en el periodo $t$. Esta medida hace la distinción entre incrementos de precio que marcan nuevos máximos en relación los 12 meses más recientes. El autor concluye que incrementos en el precio del petróleo tienen mayor impacto que las disminuciones. Además, cuando los incrementos son correcciones de disminuciones recientes, estos tienen menos poder predictivo. El enfoque ha sido reproducido también por Lee y Ni (2002).

Ferrucci, Jiménez-Rodríguez y Oronte (2010) adaptan la metodología de Mork (1989) a la medición de asimetrías en el efecto traspaso de precio de MP alimenticias en los países de la zona euro. Su estudio concluye que existen asimetrías de signo estadísticamente significativas. Además, encuentran diferencias importantes entre el traspaso a nivel agregado y a nivel de componentes de los índices de precios.

Ahora bien, Kilian y Vigfusson (2011) marcan un punto de inflexión en el estudio de las asimetrías en el ámbito de los modelos VAR. Los autores demuestran que los modelos VAR censurados, originalmente propuesto por Mork (1989), sesgan asintóticamente el efecto de precios de petróleo sobre PIB, ya sea que el proceso generador de datos (PGD) sea simétrico o asimétrico. Este resultado se mantiene aun cuando la variable de precio de energía utilizada sea una medición neta en línea con

3 Previo a estas disminuciones las series de precios de MP energéticas mostraban muy pocas variaciones negativas. 
la propuesta de Hamilton (1996). Esa inconsistencia ha sesgado la evidencia empírica documentada en favor de la hipótesis de asimetría en el traspaso del precio de las MP a precios nacionales (Bloomberg \& Harris, 1995; Cutler et al., 2005 y Ferrucci et al., 2010; Furlong \& Ingenito, 1996).

El modelo que proponen Kilian y Vigfusson (2011) genera estimadores consistentes sin importar la forma de la asimetría y con independencia de si el PGD es simétrico o asimétrico. Los autores demuestran que, aparte de generar a una estimación inconsistente de parámetros, modelos censurados conducen a una errónea cuantificación de la respuesta dinámica de la producción a choques sobre el precio de la energía. De igual forma, sugieren una manera alternativa de estimar las funciones de impulso respuesta (IR) en modelos de regresión no lineales. Esta propuesta se basa en la estimación de un modelo estructural, con lo que además de superar las limitaciones señaladas, se evita las ambigüedades sobre cómo definir un choque en el contexto de formas reducidas de modelos no lineales.

Los mismos autores proponen dos tipos de pruebas estadísticas para someter a prueba la hipótesis de simetría en la respuesta del producto a choques sobre los precios de energía. Una de las pruebas se aplica a los coeficientes de regresión, mientras la otra directamente a las funciones IR. Cabe destacar que la prueba que proponen para los coeficientes es una variante con mayor poder estadístico que lo expuesto por Mork (1989). La prueba aplicada a los IR surge del reconocimiento de que en un modelo asimétrico la respuesta dinámica de una variable puede ser estadísticamente significativa aun cuando los coeficientes no denoten alejamientos de la simetría. Alternativamente, tales respuestas pueden ser estadísticamente no significativas incluso con coeficientes estadísticamente significativos. Además, según Koop, Pesaran y Potter (1996), en modelos VAR no lineales la respuesta dinámica de las variables puede depender de la magnitud de los choques, lo cual es una limitación al tipo de prueba aplicada a los parámetros estimados pues no consideran esa posibilidad.

El presente documento analiza el traspaso de precios de MP a nivel macroeconómico y microeconómico para el caso de Costa Rica. A nivel macroeconómico, se investiga si existe evidencia de asimetrías de signo, magnitud y volatilidad. A nivel microeconómico, se estudia en qué industrias existe evidencia de asimetría de signo y si tal efecto está asociado a estructuras de organización industrial poco competitivas. El documento se organiza de la siguiente forma: en la sección metodológica se elabora el marco conceptual sobre el que se basa la aproximación empírica empleada y se describen los aspectos metodológicos más relevantes del estudio. Posteriormente, se exponen los principales resultados $y$, finalmente, se esbozan las principales conclusiones.

\section{METODOLOGÍA}

Kilian y Vigfusson (2011) proponen implementar la estimación del traspaso mediante un modelo estructural completo como el siguiente:

$$
\begin{gathered}
x_{t}=\quad a_{1} x_{t-1}+a_{2} y_{t-1}+\cdots+\epsilon_{1 t} \\
y_{t}=\beta_{1} x_{t}^{+}+\beta_{2} x_{t-1}^{+}+\beta_{3} y_{t-1}+\cdots+\epsilon_{2 t}
\end{gathered}
$$

Donde $x_{t}^{+}$denota $x_{t}$ censurada. Esto es, $x_{t}^{+}=\left\{\begin{array}{c}x_{t} \text { si } x_{t}>0 \\ 0 \text { si } x_{t} \leq 0\end{array}\right.$

El modelo estructural (1) no permite impacto contemporáneo sobre $y_{t}$ de choques negativos en $x_{t}$ Estos choques pueden tener efectos sobre la senda futura de $y_{t}$ en la medida en que conduzcan eventualmente a choques positivos en la senda futura de $x_{t}$. En contraposición, considere lo que sucede en un modelo como (2), donde el impacto contemporáneo de un choque negativo a $x_{t}$ es - $\beta_{1}$. 


$$
\begin{gathered}
x_{t}=\quad a_{1} x_{t-1}+a_{2} y_{t-1}+\cdots+\epsilon_{1 t} \\
y_{t}=\beta_{2} x_{t-1}^{+}+\beta_{3} y_{t-1}+\cdots+\beta_{1} \epsilon_{1 t}+\epsilon_{2 t}
\end{gathered}
$$

El modelo estructural (1) no puede estimarse a partir de una forma reducida como (3).

$$
\begin{aligned}
& x_{t}=a_{1} x_{t-1}+a_{2} y_{t-1}+\cdots+\epsilon_{1 t} \\
& y_{t}=\beta_{1} x_{t-1}^{+}+\beta_{2} y_{t-1}+\cdots+u_{2 t}
\end{aligned}
$$

Donde

$$
u_{2 t}=\beta_{1} \epsilon_{1 t}+\epsilon_{2 t}
$$

De (3) no es posible identificar las respectivas funciones de IR a partir de la descomposición de Cholesky de la matriz $\operatorname{Var}\left[\epsilon_{1 t}, u_{2 t}\right]$. Tal descomposición no es apropiada en vista de que $u_{2 t}$ debería reflejar, según el modelo estructural (1), solo choques positivos; sin embargo, la descomposición estándar no hace tal distinción.

\section{Estimación de impulsos respuesta en modelos VAR asimétricos}

Defínase la respuesta de $y$, condicional en sendas históricas $x_{t-i}=y_{t-i}=0$ para $i=1,2, \ldots$ de la siguiente forma:

$$
I_{v}(\delta, H, \underline{0})
$$

Si se relaja el supuesto de $x_{t-i}=y_{t-i}=0$ permitiéndose alguna historia $\left(\Omega_{\mathrm{i}}\right)$ para los choques de ambas variables, e induciendo un choque de magnitud $\delta$ en la observación t-ésima del término de perturbación $\epsilon_{1}$, se puede definir de manera alternativa la respuesta:

$$
\begin{gathered}
I_{y}^{*}\left(\delta, H, \Omega_{\mathrm{i}}\right)=E\left\{y_{t+h} \mid \Omega_{\mathrm{i}}, \epsilon_{1 t}=\delta,\left[\epsilon_{1 t+j}\right]_{j=1}^{h},\left[\epsilon_{2 t+j}\right]_{j=0}^{h}\right\} \\
-E\left\{y_{t+h}\left|\Omega_{\mathrm{i}},\left[\epsilon_{1 t+j}\right]_{j=0}^{h},\right|\right.
\end{gathered}
$$

Promediando ( 5 ) a lo largo de las posibles historias se obtiene la respuesta no condicionada en $\Omega_{\mathrm{i}}$, esto es $I_{y}^{*}(\delta, H)$.

El IR habitualmente reportado en la literatura es $I_{y}^{*}(\delta, H, \underline{0})$. Este, además de no permitir dinámica de choques futuros en los términos de perturbación, tampoco condiciona en historia alguna. Esta configuración para el cálculo del IR puede funcionar en sistemas lineales, pero en sistemas no lineales podría no converger a cero aunque el PGD sea estacionario (Koop et al., 1996). Además, es de poca utilidad obtener una estimación del IR que condiciona en 0 , debido a lo poco realista de tal condición. Finalmente, Potter (2000) favorece considerar los choques futuros como aleatorios más que fijarlos en cero cuando se estiman IR no lineales. 
Otro resultado teórico de relevancia es la relación inversa que hay entre el tamaño del choque $(\delta)$ y la importancia de considerar tanto las historias como la variabilidad de $\epsilon_{1 t}$ cuando se estiman los IR. Kilian y Vigfusson (2011) prueban que:

$$
\lim _{n \rightarrow \infty} \frac{1}{n} I_{y}(n \delta, H)=I_{y}^{*}(\delta, H, \underline{0})
$$

Es decir, para choques suficientemente grandes, la importancia de $\Omega_{\mathrm{i}}$ y la aleatoriedad de $\epsilon_{1 t}$ disminuyen hasta el punto en que el IR estimado mediante el método VAR tradicional sea una buena aproximación a la estimación correcta. Esta es, en esencia, la razón por la que el método VAR tradicional podría generar estimaciones de la reacción del precio local a choques en el precio de MP muy distintas a las correctamente estimadas mediante una especificación no lineal. Si las series de variación de precios de MP muestran bajas desviaciones estándar (lo cual es típicamente el caso al trabajar con variaciones intermensuales), la ganancia de utilizar $I_{y}(n \delta, H)$, en términos de reducción de sesgo asintótico en la medición del IR, es grande. En términos de las variables relevantes para este estudio, la propuesta de Kilian y Vigfusson (2011) requiere estimar un sistema de ecuaciones que permite a choques positivos sobre precio de MP tener un efecto diferenciado sobre los precios nacionales de aquel originado en choques negativos. En las estimaciones se utilizó un sistema compuesto por tres variables; sin embargo, para una intuición más directa y sin pérdida de generalidad, para efectos de exposición se utiliza un sistema de dos. La estructura base del modelo es la siguiente:

$$
\begin{aligned}
& x_{t}=\kappa_{1}+\quad \alpha_{11} x_{t-1}+\cdots+\alpha_{1 p} x_{t-p}+\beta_{11} y_{t-1}+\cdots+\beta_{1 p} y_{t-p}+\epsilon_{1 t} \\
& y_{t}=\kappa_{2}+\alpha_{20} x_{t}+\alpha_{21} x_{t-1}+\cdots+\alpha_{2 p} x_{t-p}+\beta_{21} y_{t-1}+\cdots+\beta_{2 p} y_{t-p}+\cdots \\
& \gamma_{20} x_{t}^{+}+\gamma_{21} x_{t-1}^{+}+\cdots+\gamma_{2 p} x_{t-p}^{+}+\epsilon_{2 t}
\end{aligned}
$$

La variable $x_{t}$ representa un indicador de precios (o de su variación) de MP y $y_{t}$ es un indicador de precios nacionales (o de su variación).

Al no permitir que cambios negativos en $x_{t}$ afecten $y_{t}$, los residuos MCO del modelo (1) pueden padecer autocorrelación. No es este el caso del modelo (7) que, por ende, puede ser estimado vía métodos de regresión estándar. Una vez estimados sus parámetros, es posible construir la respuesta dinámica de $y_{t}$ ante choques sobre $x_{t}$.

El modelo (7) anida una versión simétrica y una asimétrica del PGD. La literatura (Gallant, Rossi \& Tauchen, 1993; Koop et al., 1996) señala que en modelos no lineales la respuesta dinámica de $y_{t}$ podría ser magnificada o disminuida por el efecto acumulado de choques previos. En este sentido, los IR deberían ser calculados como el promedio de los IR generados a partir de un conjunto diverso y representativo de condiciones iniciales. En razón de esto, los IR se estimaron mediante un algoritmo que ejecuta los siguientes pasos:

i. Se estima mediante métodos estándar, los parámetros del modelo (7).

ii. Se selecciona aleatoriamente una "historia" $\left(\Omega_{i}\right)$ compuesta por una secuencia de $p$ observaciones consecutivas de $x_{t}$ y $y_{t}$.

iii. Dada $\Omega_{i}$, se simulan, tanto para $x$ como para $y$, dos sendas de $H$ datos posteriores a la última observación disponible; esto es, para $x$ se generan $\left[x_{++1}, x_{++1}, \ldots, x_{++H}\right]$ $y\left[x_{*+1}^{*}, x_{++2}^{*}, \ldots, x_{++H}^{*}\right]$; para $y$ se generan $\left[y_{t+1}, y_{t+2}, \ldots, y_{t+H}\right] y$ $\left[y_{t+1}^{*}, y_{t+2}^{*}, \ldots, y_{t+H}^{*}\right]$. Las primeras sendas de $x$ y $y$, además de la segunda de $y$, se generan a partir de las secuencias de perturbaciones estocásticas $\left[\epsilon_{1 t}, \epsilon_{1 t+1}, \ldots, \epsilon_{1 t+H}\right]$ 
$y\left[\epsilon_{2 t}, \epsilon_{2 t+1}, \ldots, \epsilon_{2 t+H}\right]$ que son seleccionadas aleatoriamente de sus respectivas distribuciones marginales empíricas. En el caso de la segunda senda de $x$, se asigna un valor $(\delta)$ al primer componente de la secuencia de perturbaciones, esto es $\epsilon_{1 t}=\delta$, mientras que el resto de la senda es extraída aleatoriamente de su distribución marginal empírica 4 .

iv. Para $t=1,2, \ldots, H$ se calcula la diferencia entre las dos sendas de $v$ - Cada elemento de la secuencia resultante es $y_{i}^{\delta}$, donde $i=1,2, \ldots H$.

v. Se efectúa cierto número $\left(n_{\text {boot }}\right)$ de repeticiones de los pasos iii y iv.

vi. Se repiten los pasos ii a $v$ cierto número $\left(n_{\text {hist }}\right)$ de veces. Con esto se tiene entonces $n_{\text {boot }} * n_{\text {hist }}$ distintas series $y_{i}^{\delta}$ que se promedian.

El promedio de los $y_{i}^{\delta}$ generado mediante los pasos iii a $v$ corresponde a la respuesta de $y$ ante un choque de tamaño $O$ sobre la serie $x$, a un horizonte de $H$ periodos y condicional en $\Omega_{i}$. Utilizando la misma nomenclatura de Kilian y Vigfusson (2011), esta respuesta será $I_{y}\left(\delta, H, \Omega_{\mathrm{i}}\right)$. Si se repite el ejercicio para una cantidad suficiente de posibles historias y se promedia las respuestas, se obtendría la respuesta de $y$ no condicionada en $\Omega$, es decir $I_{y}(O, H$.

Los IR pueden obtenerse para distintos tamaños de choque. Para asegurar comparabilidad, los promedios obtenidos en el paso vi. se reescalan dividiéndolos entre el tamaño del choque utilizado. Se trabajó con 4 tamaños de choque: 1, 2, 4 o 10 desviaciones estándar de la serie $\chi$. También se estimaron bandas de confianza empíricas para las estimaciones de IR. Estas se construyeron calculando, para cada uno de los H horizontes, los percentiles 5 y 95 de la distribución de los $n_{\text {hist }} * n_{\text {hist }}$ IR simulados.

Como se verá en la sección de resultados, las bandas de confianza de los IR muestran una tendencia a estrecharse conforme el tamaño del choque aumenta. Tal fenómeno se debe a que conforme el choque se vuelve más grande en relación a las historias, la senda del IR estimado es menos atribuible a la variedad de posibles historias, con lo cual la incertidumbre de la estimación se reduce.

\section{Pruebas de simetría}

En comparación con un VAR simétrico, el modelo ( 7 ) es asintóticamente ineficiente cuando el PGD es simétrico. De ahí que es importante disponer de alguna prueba estadística que evalúe la hipótesis de simetría del PGD. Debido a que los IR pueden ser función altamente no lineal tanto de los parámetros de pendiente como de la variancia de las innovaciones, las pruebas que evalúan igualdad de coeficientes podrían no ser efectivas para verificar el grado de asimetría en los IR de modelos estructurales. Edelstein y Kilian (2007 y 2009) sugieren someter a prueba la hipótesis de simetría mediante una aproximación alternativa basada en los IR obtenidos según el método explicado en la sección metodológica.

4 Note que es posible tratar a las secuencias aleatorias de y como independientes pues son obtenidas de la distribución marginal generada por el modelo estructural estimado (5) 


\section{Pruebas de simetría sobre parámetros}

La aproximación metodológica tradicional para someter a prueba la hipótesis de simetría en la transmisión de choques de precio de MP $\left(x_{t}\right)$ a precios nacionales $\left(y_{t}\right)$ dictaría efectuar regresiones de $y_{t}$ en sus propios rezagos $y$ los de $x_{t}^{+} y x_{t}^{-} y$ proceder con pruebas de igualdad de coeficientes haciendo uso de estadísticos de Wald (ver Mork,1989).

Kilian y Vigfusson (2011) señalan que esa aproximación no explota todas las restricciones que implica la hipótesis nula de simetría. En particular, señalan que Mork (1989), al trabajar con un modelo reducido, omite la restricción de igualdad de los términos contemporáneos de $x_{t}^{+}$y $x_{t}^{-}$. Proponen, entonces, en términos del modelo ( 7 ), trabajar con la siguiente hipótesis nula:

$$
H_{0}: \gamma_{20}=\gamma_{21}=\cdots \gamma_{2 p}
$$

Con el argumento de que tiene mayor poder que la prueba de Mork (1989), proponen someter a prueba la hipótesis (8) estimando un modelo como (7) y, por medio de pruebas de Wald de exclusión de parámetros, determinar si la inclusión de los regresores $x_{t}^{+}, x_{t-1}^{+}, \ldots, x_{t-p}^{+}$modelo.

\section{Pruebas de simetría sobre impulsos respuesta}

Los pasos de la prueba propuesta por Kilian y Vigfusson (2011) para someter a prueba la simetría de los IR se enumeran a continuación:

i. Estimar el modelo estructural ( 7 ).

ii. Calcular el IR $h$ periodos hacia adelante tanto para un choque positivo como para uno negativo. Esto es, calcular $I_{y}^{*}(\delta, h)$ y $-I_{y}^{*}(-\delta, h)$.

vii. Calcular el estadístico $W=\sum_{i=0}^{h_{0}}\left[I_{y}^{*}(\delta, i)+I_{y}^{*}(-\delta, i)\right]^{2}=\mathrm{o}$ que, bajo la hipótesis conjunta de simetría de signo en los IR hasta $h$ periodos en el futuro, $y$ dada la normalidad asintótica de los estimadores MCO del modelo (7), tiene distribución $\chi_{h+1}^{2}$.

viii. Calcular la matriz de variancias y covariancias del vector de suma de coeficientes de respuesta mediante bootstrapping.

\section{Corrección por volatilidad}

Con el objeto de determinar si existe evidencia de que el traspaso a nivel agregado muestre asimetrías en volatilidad, proponemos combinar la metodología de Kilian y Vigfusson (2011) con la propuesta de Lee et al. (1995). Esta última indica que para obtener las variaciones de precio relevantes para cuantificar el traspaso deben reescalarse las series de precio originales por algún indicador de volatilidad.

Para efectuar el reescalamiento, se estima un modelo GARCH univariado de las series de variación de precios de MP. Si $x_{t}$ es la variación mensual de precios de MP, el modelo GARCH tiene la siguiente forma general: 


$$
x_{t}=a_{0}+\sum_{i=1}^{r} a_{i} x_{t-\mathrm{i}}+\sum_{i=1}^{s} \beta_{i} z_{t-\mathrm{i}}+\epsilon_{t}
$$

Donde $\left\{z_{t-j}: j \geq 1\right\}$ representa variables relevantes contenidas en el conjunto de información $I_{t-1}, t=1,2, \ldots T$ y $\beta$ es el respectivo vector de coeficientes. Igualmente $\epsilon_{t} \mid I_{t-1} \backsim N\left(0, \sigma_{t}^{2}\right)$ $y$, como suele ser estándar en los modelos de heteroscedasticidad condicional autoregresiva

$$
\sigma_{t}^{2}=\gamma_{0}+\sum_{i=1}^{q} \gamma_{i} \epsilon_{t-i}^{2}+\sum_{j=1}^{p} \gamma_{q+j} \sigma_{t-j}^{2}
$$

Definida de esta forma, $\epsilon_{t}$ es la parte inesperada de la variación en el precio de la MP cuyo impacto sobre precios nacionales es de interés; sin embargo, $\epsilon_{t}$ no incorpora alteraciones en la variabilidad condicional de $x_{t}$.

El argumento de Lee et al. (1995) es que si el PGD está adecuadamente descrito por ( 9 ), una medida que refleja tanto la magnitud como la volatilidad de $\epsilon_{t}$ es la siguiente:

$$
\epsilon_{t}^{*}=\frac{\epsilon_{t}}{\sqrt{\sigma_{t}^{2}}}
$$

Según los autores, $\epsilon_{t}^{*}$ debería tener un efecto más significativo y sistemático que $x_{t}$ y que $\epsilon_{t}$ , sobre la variación de precios nacionales. Dentro de los resultados que se muestran más adelante, se contrasta el traspaso estimado haciendo uso del indicador $\epsilon_{t}^{*}$ de (10) con aquellos que no consideran tal corrección por volatilidad.

\section{Datos}

Los precios de MP utilizados corresponden a índices de precio mensuales $(2010=100)$ publicados (Banco Mundial, 2016). Para el análisis de traspaso a nivel agregado (macro) se utilizaron dos índices: el correspondiente a Energía (Energy), que pondera petróleo crudo (84,6\%), gas natural (10,8\%) y carbón (4,6\%); y el denominado índice de MP no energéticas (Non-Energy), que pondera productos agrícolas $(64,9 \%)$, metales $(31,6 \%)$ y fertilizantes $(3,5 \%)$. Los ponderadores de las agrupaciones corresponden al peso relativo que los respectivos componentes tuvieron en el valor de exportación de países en desarrollo entre 2002 y 2004.

Para el caso del análisis desagregado a nivel de industrias (micro), se utilizaron los índices de precio de trigo, maíz, soya, aceite de palma, arroz, azúcar, café, tabaco, caucho, cobre, aluminio, hierro, fertilizantes, polímeros de plástico y el precio del barril de petróleo West Texas Intermediate.

Todos los índices de precios de MP están disponibles desde enero de 1960 y se utilizaron datos hasta marzo de 2016 para el caso del análisis a nivel macro; $y$ hasta diciembre 2015, a nivel micro.

Las series de precios nacionales provienen de diversas fuentes. Para el análisis agregado, se utilizó el IPC base junio 2015. Esta serie se obtuvo con frecuencia mensual desde enero de 1976 en Banco Central de Costa Rica [BCCR] (2016a). Para el caso del análisis a nivel micro, se trabajó con un total de 80 productos; de estos, 56 son componentes del IPC, obtenidos de Instituto Nacional de Estadística y Censos [INEC] (2016), y 24 del Índice de Precios al Productor de la Manufactura (IPPMAN). Estas últimas series son elaboradas por el Banco Central de Costa Rica; sin embargo, por razones de confidencialidad solo el indicador agregado se publica

En los modelos estimados se incluyó, además de series de precio de MP y precios nacionales, el TC (colones/dólar) promedio de venta del mes, cuya serie está disponible en BCCR (2016b). Esta inclusión es relevante en una economía donde la mayoría de precios nacionales se fijan en moneda local y, por ende, debe controlarse por TC para identificar del traspaso de precios de MP a precios locales. 


\section{Resultados}

Al estudiar las propiedades estacionarias de los índices de precios de MP y de productos domésticos, se concluye que son variables integradas de orden uno [I(1)] que no cointegran ${ }^{5}$. Según esto, se descartó una aproximación metodológica de corrección de errores y se procedió a utilizar los índices de precios en su primera diferencia logarítmica.

GRÁFICO 1.

SUMA DE COEFICIENTES DE REZAGOS DE MATERIAS PRIMAS ENERGÉTICAS EN ECUACIÓN DE INFLACIÓN

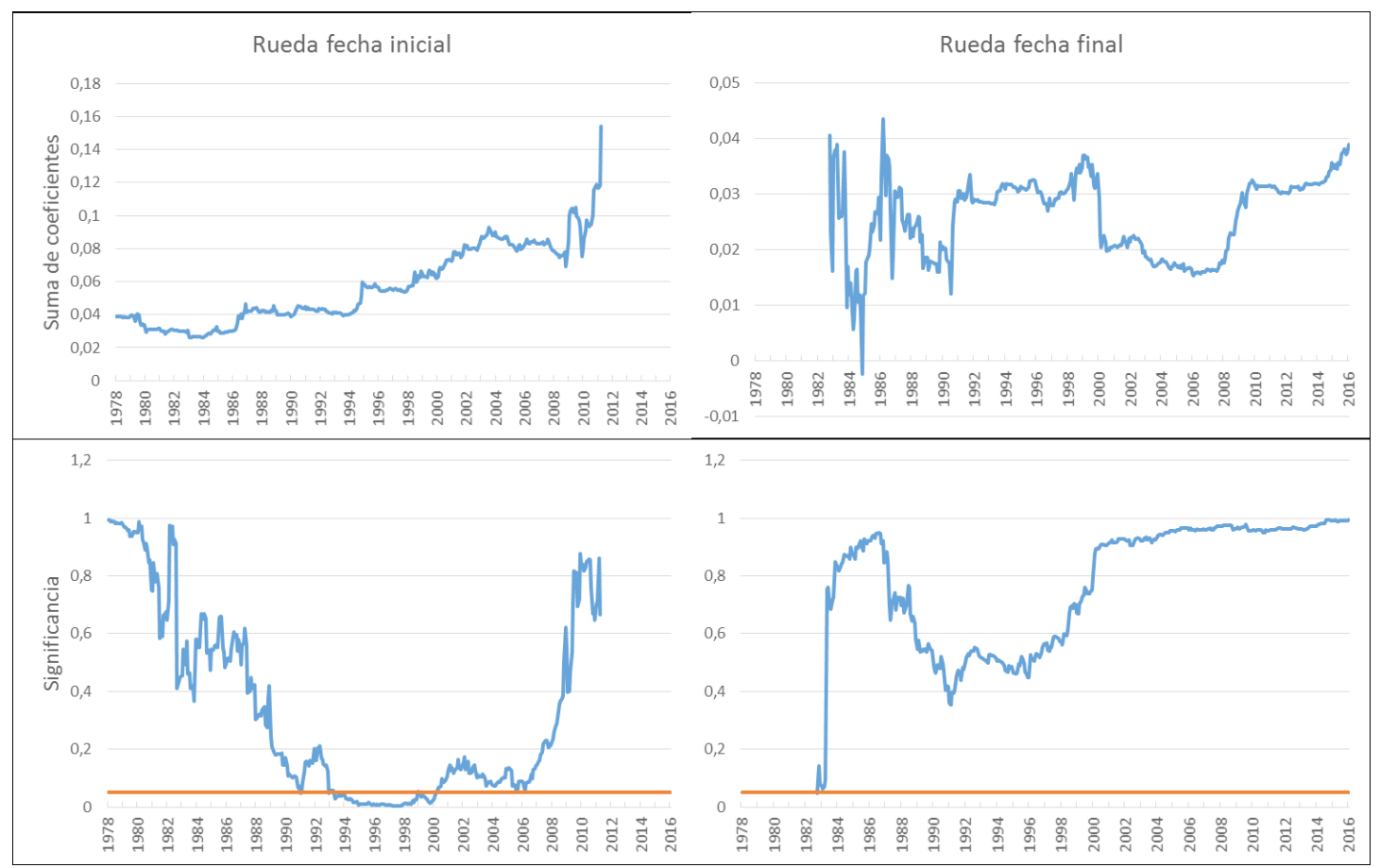

Fuente: Elaboración propia

\section{Traspaso a nivel macro}

\section{Estabilidad de la relación entre precios nacionales y de MP}

Se estimaron dos conjuntos de modelos VAR con muestras rodantes ${ }^{6}$, uno con precios de MP energéticas y otro con MP no energéticas. En todos los casos, se consideró hasta 12 rezagos y las variables endógenas fueron: índice de precios MP, IPC y TC. El gráfico 1 muestra la suma de

6 Se implementaron dos formas de hacer "rodar" la muestra: En "Rueda fecha inicial" se parte de una muestra "grande" de 456 observaciones (marzo-1978 a marzo-2016) y secuencialmente se va excluyendo una observación a la vez al inicio de muestra. El proceso se repite 400 veces con lo que el modelo con menos datos abarca de junio 2011 a marzo 2016 (45 observaciones). En el caso de "Rueda fecha final", se parte de una muestra "pequeña" de 45 observaciones (marzo-1978 a diciembre-1982) y secuencialmente se añade una observación al final de la muestra. De igual forma el proceso se repite 400 veces hasta que el modelo con más datos abarca de marzo-1978 a marzo-2016 (456 observaciones). 
coeficientes de los rezagos del indicador de precios de MP energéticas en la ecuación de inflación y el valor de su significancia estadística conjunta. La línea horizontal en el nivel 0,05 señala el 5\% de significancia. En los gráficos de la izquierda (Rueda fecha inicial), cada punto corresponde a los resultados de la estimación que utiliza información desde esa fecha hasta marzo de 2016. En los gráficos de la derecha (Rueda fecha final) cada punto corresponde a la estimación con datos desde marzo de 1978 hasta ese momento.

La exclusión progresiva de observaciones anteriores a la primera mitad de la década de los noventa incrementa la significancia conjunta de coeficientes al tiempo que la magnitud de su suma mantiene tendencia creciente. Según estos resultados, mantener observaciones anteriores a la década de los noventa impide alcanzar una relación econométrica estadísticamente significativa entre precios de MP energéticas e IPC. En conclusión, la relación entre el precio de MP y el IPC no ha sido estable en el tiempo. Fue sumamente débil previo a la primera mitad de la década de los noventa y se ha fortalecido desde entonces. Para el caso de los modelos VAR estimados con el indicador de precios de MP no energéticas, el resultado es similar.

Este ejercicio de estimación con muestras rodantes es útil no solo para analizar la evolución de la relación entre los índices de precio de MP con el IPC, además orienta la elección de la muestra que se utiliza en la aplicación de la metodología propuesta por Kilian y Vigfusson (2011) para investigar la existencia de efectos asimétricos. La muestra de datos finalmente utilizada para el análisis de asimetrías en el traspaso a nivel macro abarca, para el caso de MP energéticas, desde enero de 1995 a marzo 2016; y entre enero de 1991 y marzo 2016 para las estimaciones del traspaso de MP no energéticas. Además, con esas muestras se determinó que el orden óptimo de rezagos en los modelos VAR es 3 y 6 respectivamente. Se efectuaron pruebas de causalidad en el sentido de Granger cuyos resultados (ver Cuadro 1 del Anexo.) sugieren, como es esperable, que los movimientos de precios de las MP anteceden a los del IPC.

\section{Pruebas de asimetría}

De acuerdo con el cuadro 2 del anexo, que muestra los resultados de aplicar la prueba de simetría sobre parámetros explicada en la sección metodológica, no es posible rechazar las hipótesis de traspaso simétrico para cambios de precios de MP energéticas ni de MP no energéticas. La conclusión es la misma aplicando la versión modificada de la prueba propuesta por Mork (1989).

Ahora bien, si el PGD es asimétrico los IR pueden ser función altamente no lineal tanto de los parámetros de pendiente como de la variancia de las innovaciones. Así que aun cuando no se encuentre evidencia de asimetría de signo en los parámetros, los IR ante choques positivos pueden ser muy diferentes respecto a los generados con choques negativos. De ahí la recomendación de aplicar pruebas de simetría directamente a los IR estimados. El cuadro 3 del anexo muestra los resultados de las pruebas de simetría aplicadas a los IR, según se expone en la sección metodológica. Estos resultados se obtuvieron a partir de 250.000 simulaciones de un modelo como (7) ampliado para incluir el TC como variable endógena, con tres y seis rezagos respectivamente para los casos de índices de precios de energía y no energía, y con hasta 24 periodos de pronóstico. Además, en vista de la posible asimetría al tamaño de los choques, se muestran resultados para choques de una y dos desviaciones estándar de las innovaciones. La evidencia en contra de la simetría es escasa; únicamente, para el caso de precios de MP energéticas a horizontes menores a tres meses y si se admite una tolerancia del 10\% al error estadístico tipo 1, se rechaza la hipótesis de simetría. Para horizontes más largos tal evidencia se debilita sustancialmente en todos los casos. 


\section{Traspaso de precios de MP a IPC}

Los resultados que se muestran a continuación provienen de modelos estructurales completos que anidan un PGD simétrico y asimétrico según Kilian y Vigfusson (2011). Se cuantifica el traspaso de precios de MP al IPC considerando tres posibles tipos de asimetría: de signo, de magnitud y de volatilidad. Todas las funciones de IR que se presentan, se generaron a partir de 250.000 estimaciones $^{7}$. Son funciones de IR acumuladas del IPC ante choques sobre el respectivo índice de precios de MP. Para facilitar la comparación, las funciones IR asociadas a choques negativos aparecen multiplicadas por -1.

En primera instancia, y para efectos de contrastar la estimación que resulta de aplicar la metodología de Kilian y Vigfusson (2011) con la de un VAR censurado tradicional, según se aprecia en el gráfico 2, para el caso de precios de energía el VAR censurado tradicional sobreestima la respuesta dinámica del IPC para los 3 tamaños de choque más grandes. Nótese además que la estimación puntual indica que la respuesta del IPC es de menor magnitud proporcional conforme los choques se hacen más grandes ${ }^{8}$. Para el caso de precios de MP no energéticas, la subestimación del VAR censurado se da para todos los tamaños de choque. Al igual que en el caso anterior, la respuesta dinámica del IPC decrece con el tamaño del choque. Según Koop et al. (1996), la dirección del sesgo asintótico es ambigua, así que tanto sobreestimaciones o subestimaciones son esperables.

En el gráfico 3 es posible comprar la respuesta dinámica del IPC a choques positivos y negativos sobre precios de MP energéticas y no energéticas. La estimación puntual indica que, para todos los tamaños de choque, la respuesta es mayor cuando se trata de choques negativos. Además, tal diferencia es creciente en el tamaño del choque. Este es un resultado que contrasta con la percepción popular de que los choques positivos, sobre todo a combustibles, se trasmiten en mayor magnitud a la generalidad de precios de la economía costarricense. Nótese sin embargo que, según las bandas de confianza empíricas, la diferencia en la respuesta es estadísticamente significativa solo para el caso de choques "grandes" (4 y 10 desviaciones estándar).

Para el caso de choques positivos y negativos sobre el precio de MP no energéticas, la estimación puntual indica que los choques negativos se transmiten más que los positivos y la diferencia es mayor cuanto más grande sea el choque. Antes del sexto mes y para los choques de 2, 4 y 10 desviaciones estándar, la respuesta a choques positivos es ligeramente mayor. Sin embargo, según las bandas de confianza, estas diferencias no son estadísticamente significativas a ningún horizonte y para casi todos los tamaños de choque. La excepción es la respuesta al choque de 10 desviaciones estándar luego de 24 meses, caso que muestra una magnitud mayor en la respuesta al choque negativo.

\section{Asimetrías por volatilidad}

Se procedió a cuantificar el traspaso reescalando las series de variaciones de precio de MP, según se explica en el apartado metodológico. Las series utilizadas para reescalar corresponden a la variancia condicional de un modelo ARMA $(1,0)$ con esquemas de residuos GARCH $(1,1)$ para el caso de MP no energéticas, y GARCH $(3,0)$ para las MP energéticas ${ }^{9}$.

7 Se seleccionó $n_{\text {boot }}=500 n_{\text {boot }}=500$ y $n_{\text {hist }}=500 n_{\text {hist }}=500$. Esto es 500 distintas historias para basar pronóstico y, para cada una de ellas, 500 simulaciones distintas del modelo (7) para cada horizonte de pronóstico.

8 Las bandas de confianza empíricas no indica diferencia estadísticamente significativa entre las respuestas a las distintas magnitudes de choque.

9 Las pruebas y resultados para la selección del mejor modelo GARCH están a disposición del lector que las solicite. 
GRÁFICO 2

IMPULSO RESPUESTA ACUMULADO DEL IPC, VAR CENSURADO VS. MODELO ESTRUCTURAL*

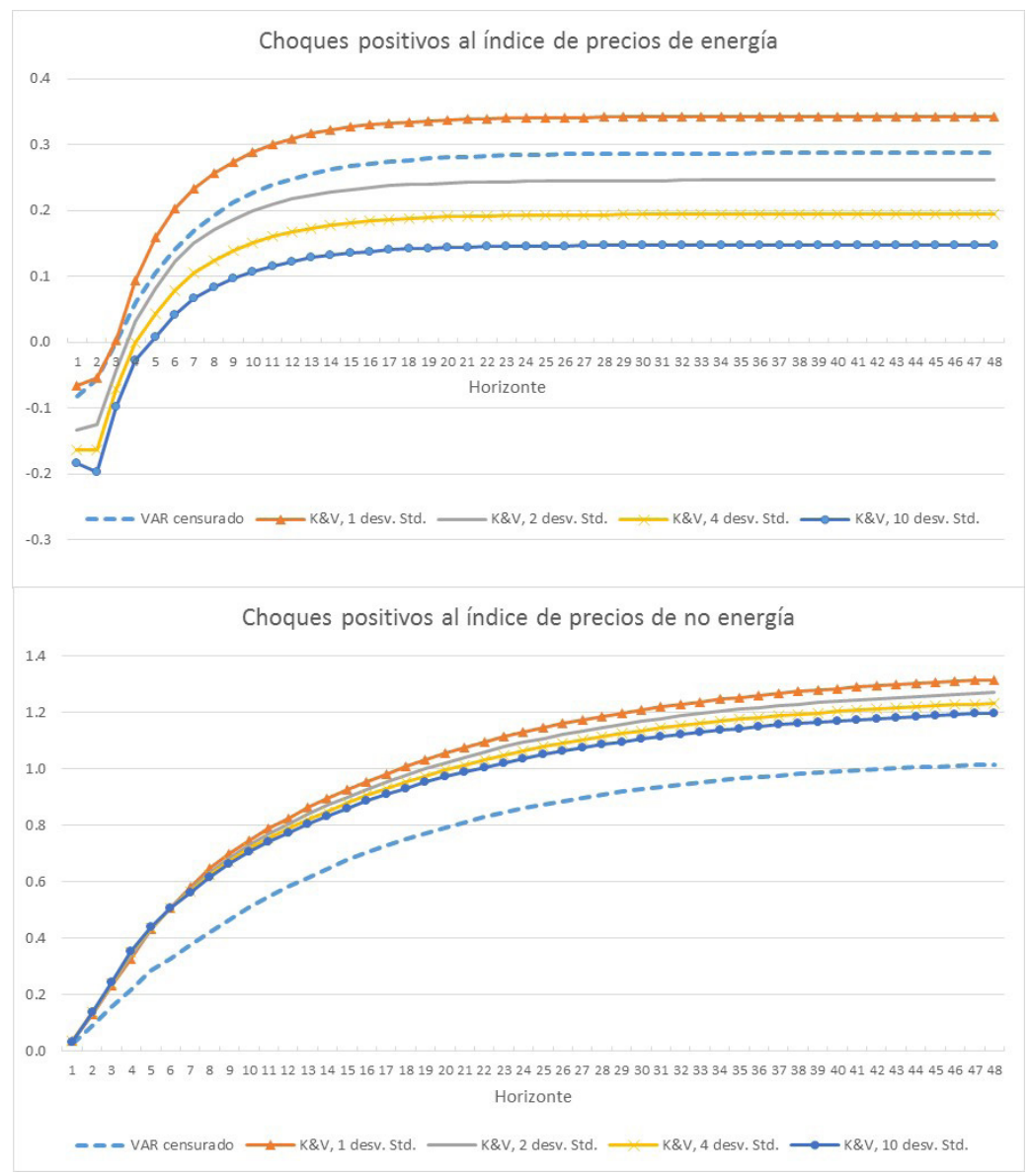

* Los IR son reescalados según el tamaño del choque para asegurar comparabilidad Fuente: Elaboración propia

Con las series de variación mensual de precios reescaladas, se procedió a corroborar la presencia de asimetrías en los parámetros mediante las pruebas propuestas por Mork (1989) y la modificación propuesta por Kilian y Vigfusson (2011). Los resultados se muestran en el cuadro 4 del anexo. La conclusión respecto a la presencia de asimetrías de signo no difiere de la obtenida para el caso sin reescalar, no es posible rechazar la hipótesis de simetría.

Igualmente, se sometió a prueba la hipótesis de simetría en las funciones IR con las estimaciones generadas a partir de las series de precio reescaladas. Los resultados se muestran en el cuadro 5 del anexo. En general estos no difieren sustancialmente de aquellos obtenidos para el caso de precios sin reescalar por volatilidad. Únicamente, para precios de MP energéticas y para un horizonte de un mes se reporta evidencia significativa (al 10\%) de asimetría de signo. 
GRÁFICO 3

IMPULSO RESPUESTA ACUMULADO DEL IPC Y BANDAS DE CONFIANZA EMPÍRICAS CHOQUES SOBRE PRECIOS DE MP ENERGÉTICAS
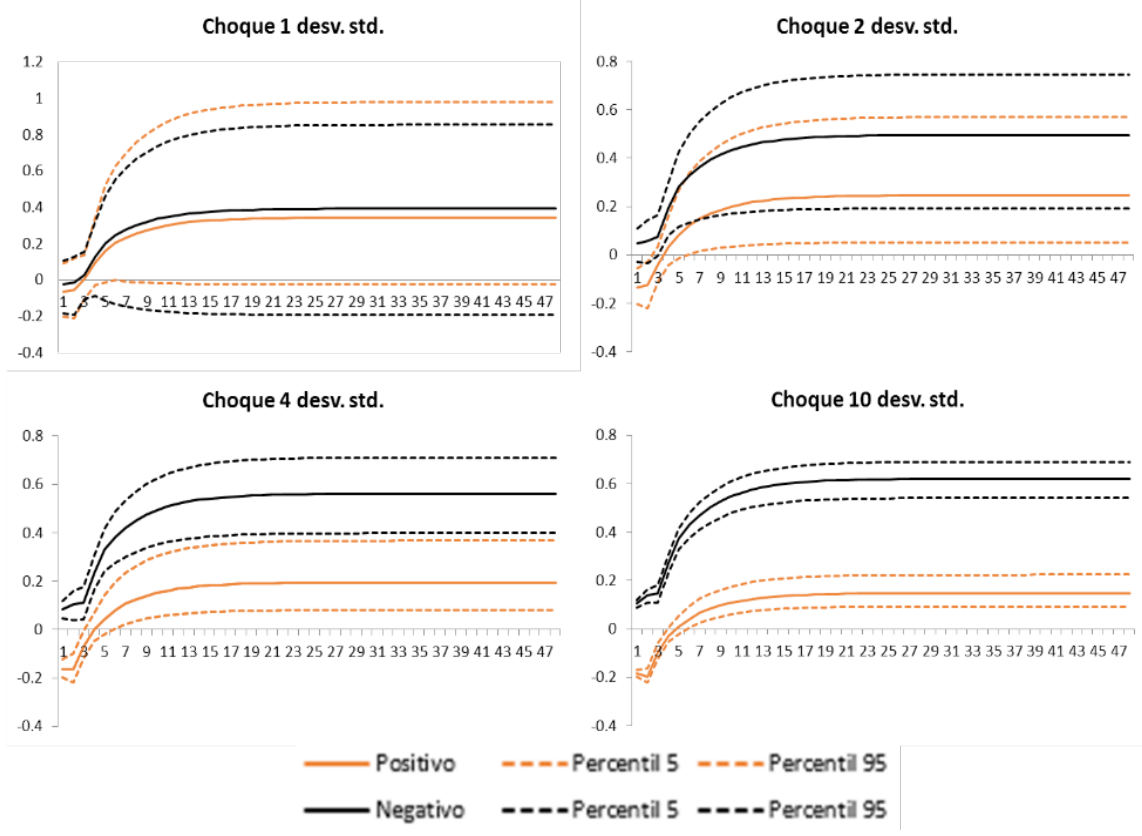

CHOQUES SOBRE PRECIOS DE MP NO ENERGÉTICAS

Choque 1 desv. std.

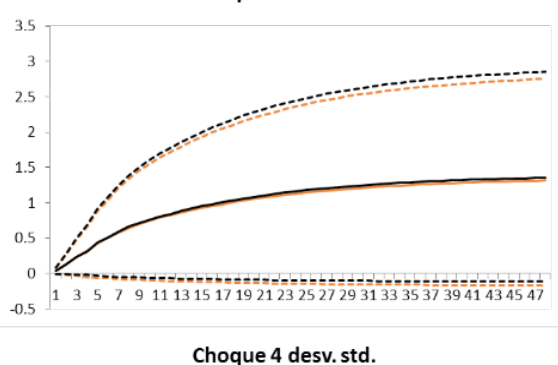

Choque 4 desv. std.

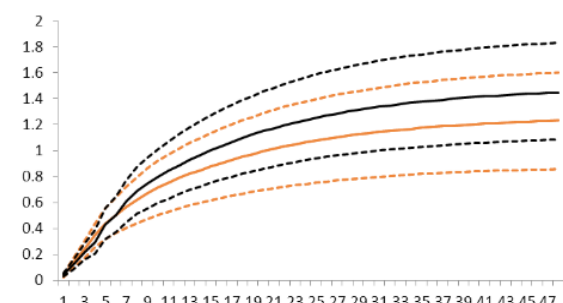

$135 \quad 7 \quad 911131517192123252729313335373941434547$

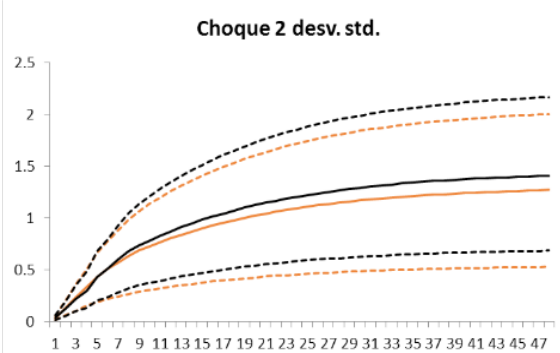

Choque 10 desv. std.

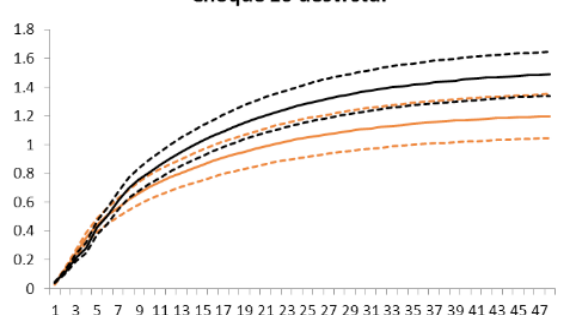

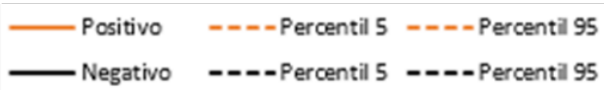

Fuente: Elaboración propia 
Los resultados que se ilustran en el gráfico 4 permiten comparar la respuesta del IPC, con y sin corrección por volatilidad, para el caso de choques positivos sobre precios de MP energéticas (en el gráfico 6 del anexo se muestra el caso equivalente para choques negativos). Para todos los tamaños de choque considerados (tanto positivos como negativos) los intervalos de confianza de las respuestas dinámicas con y sin control por volatilidad se traslapan. Se concluye, entonces, que la volatilidad del precio de las MP energéticas ( $y$ no energéticas) no condiciona la magnitud ni la simetría del traspaso identificada en la sección anterior.

\section{GRÁFICO 3 \\ IMPULSO RESPUESTA ACUMULADO DEL IPC Y BANDAS DE CONFIANZA EMPÍRICAS CHOQUES SOBRE PRECIOS DE MP ENERGÉTICAS}

Choque 1 desv. estd.

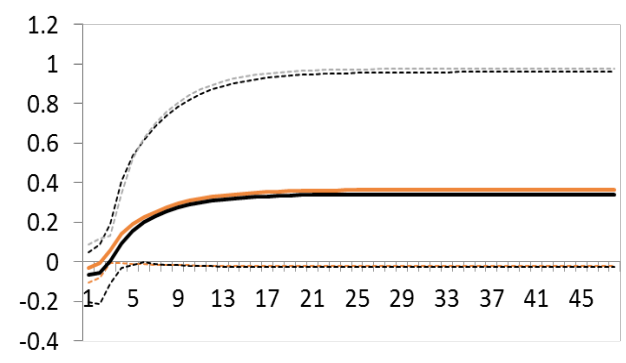

Choque 4 desv. estd.

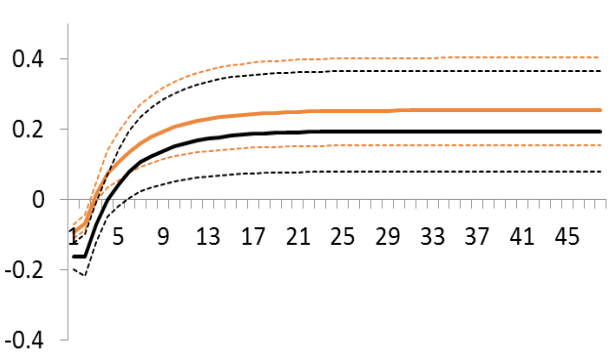

- Con corrección por volatilidad

- Sin corrección por volatilidad
Choque 2 desv. estd.

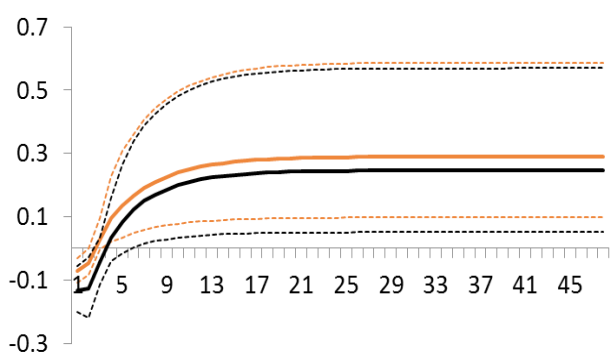

Choque 10 desv. estd.

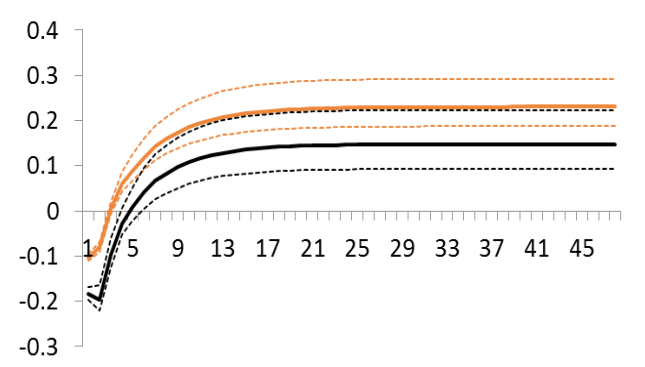

........ Intervalo del $90 \%$
Intervalo del $90 \%$

Fuente: Elaboración propia

Si bien no se encuentra un traspaso estadísticamente diferente, la estimación puntual señala un traspaso mayor cuando se considera la volatilidad del precio de las MP. Además, esta diferencia es relativamente mayor cuando el choque aplicado es más grande. Esto es coherente con la hipótesis de que los ajustes a precios locales son proporcionalmente menores ante choques sobre MP, cuando estos se presentan en contextos de alta volatilidad (ver Lee et al., 1995).

El caso para choques sobre el precio de MP no energéticas es similar, no hay diferencia estadísticamente significativa (ver gráficos 7 y 8 del anexo). De igual forma, las estimaciones centrales también señalan una respuesta ligeramente mayor cuando se controla por volatilidad. 


\section{Traspaso a nivel microeconómico}

\section{Pruebas de simetría en los impulsos respuesta}

Los resultados que se muestran a continuación se obtuvieron a partir del mismo proceso de estimación descrito en el apartado metodológico. Se estiman modelos estructurales que incorporan tres variables endógenas en primera diferencia logarítmica ${ }^{10}$ : índice de precio internacional de MP, TC nominal e índice de precio de producto local con que está relacionado (que la utiliza como insumo importante) la respectiva MP. Se seleccionó la cantidad de rezagos a partir de pruebas de exclusión ${ }^{11}$. Las pruebas de simetría se aplican sobre en los IR a partir de 250.000 simulaciones en cada caso.

Se halló evidencia de asimetría de signo (al 5\% de significancia) en 16 de los 80 casos analizados. El cuadro 6 en el anexo muestra, para esos 16 productos y para cada uno de los horizontes desde 1 hasta 24 meses, los resultados de las pruebas aplicadas. De acuerdo con estos, la asimetría de signo en el traspaso se concentra en tres grupos de bienes:

- Bienes que utilizan el trigo como insumo importante: harina y subproductos de harina (IPPMan), macarrones y fideos(IPP-Man), pan cuadrado(IPC), pastas (IPC), harina de trigo (IPC).

- Bienes que utilizan maíz como insumo importante: alimento para ganado (IPP-Man), pollo y productos de pollo (IPP-Man), pechuga de pollo (IPC), pollo entero (IPC), alas de pollo (IPC).

- Bienes que utilizan arroz como insumo importante: arroz (IPP-Man), arroz (IPC).

De los 16 productos para los que se reporta evidencia de asimetría, 13 presentan una respuesta dinámica del precio local más pronunciada ante incrementos en el precio de la MP que ante disminuciones. Además, para este grupo de bienes la asimetría se da tanto a nivel de precios al productor como de precios al consumidor. Esto hace pensar que la asimetría en el ajuste de los precios al productor puede estarse transmitiendo a lo largo de la cadena productiva hasta afectar los precios al consumidor.

Los gráficos del 9 al 12 en el anexo muestran las funciones de IR estimadas para cuatro de los 16 productos que presentan evidencia de asimetría de signo. Estas funciones se presentan reescaladas por el tamaño del choque $y$, en el caso de choques negativos, multiplicadas por - 1 . Tres de los productos evidencian asimetría positiva (harina y subproductos de harina, pollo y productos de pollo y arroz) y uno asimetría negativa (hierro y productos de hierro). Aparte de la asimetría positiva, para estos tres casos la estimación puntual de los IR señala que los oferentes no solo trasmiten más los incrementos de precio, sino que, cuando estos incrementos son grandes, los trasmiten más que cuando son pequeños $y$, de igual forma, cuando el precio de la MP disminuye, transmiten menos en esa baja entre mayor sea la disminución. Esta dinámica, claramente perjudicial para los demandantes, sería esperable en mercados donde los oferentes enfrentan poca o ninguna competencia.

Caso especial lo constituye el arroz. Nótese cómo, según se muestra en la gráfico 11, las funciones de IR ante choques negativos terminan en un nivel inferior a cero. Esto implica que, ante disminuciones del precio de la MP, la respuesta dinámica del precio local es un incremento. Esto puede estar reflejando la ausencia total de mecanismos de mercado funcionando en razón de ser este un precio regulado por el Ministerio de Economía. El razonamiento teórico para respaldar esa regulación sería evitar la extracción de rentas monopólicas en perjuicio del consumidor. Sin embargo, es claro que termina imprimiendo, desde la perspectiva del consumidor, la peor dinámica de ajuste de precios internos ante movimientos del precio internacional de la MP.

$7 \quad$ Se trata de series I(1) que no cointegran. Por razones de espacio no se muestran las pruebas respectivas, pero sus resultados están disposición de quien los solicite.

8 Se utilizaron pruebas tipo Wald sobre la significancia conjunta de los rezagos de todas las variables endógenas a cada longitud de rezago. 
Vale la pena mencionar el caso de los combustibles que, al igual que el arroz, es un bien con precio regulado. Para estos, no se encuentra evidencia de asimetría. Este es el resultado que se esperaría para un precio que por ley debe fijarse al costo según una fórmula predefinida que activa ajustes automáticos según la evolución del precio de la MP. Este método de fijación tarifaria no induce un sesgo a que el precio responda más ante aumentos o reducciones en el precio internacional del petróleo. En contraposición, la fijación del precio del arroz, cuyo método de ajuste no es automático, propicia la dinámica comentada anteriormente.

Cabe resaltar que no se encontró diferencia significativa en el tiempo que tardan los choques en el precio de petróleo en transmitirse hacia los precios finales de la gasolina y el diésel. Las funciones de IR de estos productos indican que alrededor que un $80 \%$ del efecto final se transmite en 3 meses tanto para choques positivos como negativos. El cuadro 7 del anexo muestra un resumen de los efectos acumulados para cada uno de los productos en los que se encontró evidencia de asimetría.

\section{Relación entre asimetría en el traspaso y nivel de competencia}

La asimetría en el traspaso hallada podría deberse a la existencia de estructuras de mercado poco competitivas generadas por alta concentración de oferentes. Con el objetivo de verificar esta hipótesis se estimó, para los productos con evidencia de asimetría, la relación entre un indicador de concentración de mercado y uno del nivel de asimetría en el traspaso.

$\mathrm{El}$ indicador de concentración corresponde al índice de Herfindahl- Hirshman (IHH) ${ }^{12}$. El de asimetría es:

$$
A=\frac{I R_{48}^{+}-I R_{48}^{-}}{\text {Choque }},
$$

donde

$I R_{48}^{+}$es el nivel acumulado del IR ante un choque positivo luego 48 periodos,

$I R_{48}^{-}$es el equivalente para choques negativos $y$

Choque es la magnitud del choque que genera los IR.

En el gráfico 5 se muestran los pares ordenados de los indicadores de concentración y asimetría. En el gráfico de la izquierda se incluyen todos los productos en los que se encontró evidencia de asimetría de signo, mientras que en el de la derecha se excluye los dos casos de arroz (IPP-Man e IPC). La línea punteada representa un ajuste de mínimos cuadrados. Se hace la distinción de los casos con arroz y sin él en vista de que en Costa Rica, si bien hay un número importante de industriales del arroz (lo que genera un IHH reducido), estos se comportan como un cartel, pues la mayoría son miembros de la Corporación Arrocera Nacional (Conarroz). Por ley, Conarroz posee el monopolio de la importación del producto en granza y, desde el año 2002, lo puede ingresar al país libre de impuestos. Aunado a esto, el arroz se mantiene como uno de los productos cuyo precio es regulado. Así que nada obliga a los arroceros a trasladar al consumidor eventuales reducciones del precio internacional. Como es de esperar, este mecanismo ha permitido a los productores e industriales extraer rentas de los consumidores que en ausencia de la regulación de precio no habrían obtenido ${ }^{13}$. Estos elementos hacen pensar que la medición de la relación entre concentración y nivel de asimetría estaría distorsionada en caso de incluirse el arroz.

12 Petrecolla (2006) calcula que para el periodo 1995-2005 el monto de estas rentas alcanzó 396 millones de dólares. 
Según se ilustra en el gráfico 5, la relación entre concentración y nivel de asimetría, si bien es positiva, es muy débil en magnitud y significancia estadística cuando se incluyen todos los productos. Mientras que cuando se excluyen los dos productos de arroz, esa relación positiva se fortalece tanto en magnitud como en significancia estadística.

\section{GRÁFICO 5 \\ GRADO DE ASIMETRÍA EN EL TRASPASO VS CONCENTRACIÓN DE OFERENTES}
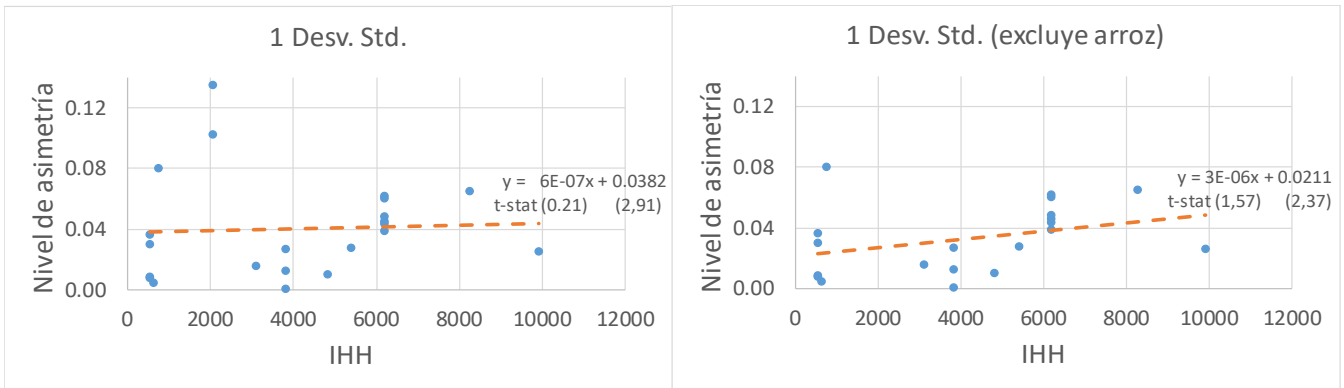

Fuente: Elaboración propia

\section{Conclusiones}

La relación dinámica del IPC con los precios internacionales de materias primas no ha sido estable en Costa Rica a lo largo de los últimos 40 años. El nivel de traspaso se ha fortalecido en magnitud y significancia estadística.

La respuesta del IPC ante choques pequeños sobre el precio de las materias primas muestra escasa evidencia de asimetría de signo. Se encontró asimetría (negativa) estadísticamente significativa solo ante choques sobre materias primas energéticas y en el muy corto plazo (3 meses o menos).

La respuesta del IPC ante choques grandes sobre precios de materias primas es asimétrica. Las disminuciones de precios de materias primas energéticas y no energéticas se traspasan en mayor cuantía al IPC que los incrementos.

La volatilidad en el precio de las materias primas no condiciona la evidencia hallada sobre asimetrías en el traspaso. Si bien la estimación puntual de la magnitud del traspaso es mayor cuando los precios de materias primas son estables, la diferencia respecto a situaciones de precios volátiles no es estadísticamente significativa.

A nivel desagregado existe evidencia de asimetría en el traspaso en 16 de los 80 productos analizados. En la mayoría de estos casos la asimetría es positiva.

Los grupos de productos donde existe evidencia de asimetría positiva están asociados a materias primas como trigo, maíz y arroz. En estos casos, la asimetría se presenta tanto a nivel de precios al productor como del consumidor. No se descarta, entonces, que la asimetría a nivel de los precios al productor se esté transmitiendo a lo largo de la cadena productiva hasta afectar los precios al consumidor.

Mayores niveles de concentración de mercado se asocian con un grado mayor de asimetría positiva en el traspaso. Como implicación de política se recomienda que la autoridad competente en materia de competencia revise a profundidad la estructura de organización industrial, así como las prácticas comerciales de las empresas dominantes en los mercados donde se encontró evidencia de asimetría positiva conjunto con alta concentración de oferentes. En segundo término, en vista del resultado observado en el caso del arroz, cuya dinámica de ajuste ha sido sumamente perjudicial para el consumidor, antes de optar por regulación de precios en industrias monopólicas, se recomienda adoptar políticas que levanten barreras de acceso a otros oferentes. 


\section{v. Referencias}

Banco Mundial (2016). Commodity Markets. Washington DC, USA: The World Bank Group. Recuperado de http://www.worldbank.org/en/research/commodity-markets.

BCCR (2016a). Índices de Precios. San José, Costa Rica: Banco Central de Costa Rica. Recuperado de http://www.bccr.fi.cr/indicadores_economicos_/Indices_Precios.html

BCCR (2016b). Tipos de Cambio. San José, Costa Rica: Banco Central de Costa Rica. Recuperado de http://www.bccr.fi.cr/indicadores_economicos_/Tipos_cambio.html

Bernanke, B.; Gertler, M. \& Watson, M. (1997). Systematic Monetary Policy and the Effects of Oil Price Shocks. Brookings Papers on Economic Activity, 1, 91-157. Recuperado de https://www. brookings.edu/bpea-articles/systematic-monetary-policy-and-the-effects-of-oil-price-shocks/

Bloomberg, S. B. \& Harris, E. S. (1995). The Commodity-Consumer Prices Connection: Fact of Fable? Federal Reserve Bank of New York Economic Policy Review, 1(3) 21-38.

Cutler, J., Chan, C. \& Li, U. (2005). The Relationship Between Commodity and Consumer Prices in Mainland China and Hong Kong. Hong Kong: Monetary Authority Quarterly Bulletin.

Edelstein, P., \& Kilian, L. (2009). How sensitive are consumer expenditures to retail energy prices? Journal of Monetary Economics, 56(6), 766-779. https://doi.org/10.1016/j.jmoneco.2009.06.001

Edelstein, P., \& Kilian, L. (2007). The Response of Business Fixed Investment to Changes in Energy Prices: A Test of Some Hypotheses about the Transmission of Energy Price Shocks. The B.E. Journal of Macroeconomics, 7(1). https://doi.org/10.2202/1935-1690.1607

Ferrucci, G., Jiménez-Rodríguez, R. \& Oronte, L. (2010). Food Price Pass-Through in the Euro Area, the Role of Asymmetries and Non-Linearities. Working Paper Series No 1168. European Central Bank.

Furlong, F. \& Ingenito, R. (1996). Commodity Prices and Inflation. FRBSF Economic Review, 2, 2747. Recuperado de https://www.frbsf.org/economic-research/files/furlong.pdf

Gallant, A. R., Rossi, P. E. \& Tauchen, G. (1993). Nonlinear Dynamic Structures. Econometrica, 61(4), 871-907. https://doi.org/10.2307/2951766

Hamilton, J. D. (1996). This is what happened to the oil price-macroeconomy relationship. Journal of Monetary Economics, 38(2), 215-220. https://doi.org/10.1016/S0304-3932(96)01282-2

Hamilton, J. D. (2003). What is an oil shock? Journal of Econometrics, 113(2), 363-398. https://doi. org/10.1016/S0304-4076(02)00207-5

INEC (2016). Índice de precios al consumidor. San José, Costa Rica. Instituto Nacional de Estadística y Censos. Recuperado de http://www.inec.go.cr/economia/indice-de-precios-al-consumidor

Kilian, L., \& Vigfusson, R. J. (2011). Are the responses of the U.S. economy asymmetric in energy price increases and decreases?: Are responses of the U.S. economy asymmetric? Quantitative Economics, 2(3), 419-453. https://doi.org/10.3982/QE99

Koop, G., Pesaran, M. H., \& Potter, S. M. (1996). Impulse response analysis in nonlinear multivariate models. Journal of Econometrics, 74(1), 119-147. https://doi.org/10.1016/0304-4076(95)01753-4

Lee, K., \& Ni, S. (2002). On the dynamic effects of oil price shocks: a study using industry level data. Journal of Monetary Economics, 49(4), 823-852. https://doi.org/10.1016/S0304-3932(02)00114-9

Lee, K., Ni, S. \& Ratti, R. A. (1995). Oils Shocks and the Macroeconomy: The Role of Price Volatility. The Energy Journal, 16(4), 39-56. https://doi.org/10.5547/ISSN0195-6574-EJ-Vol16-No4-2

Mork, K. A. (1989). Oil and the Macroeconomy When Prices Go Up and Down: An Extension of Hamilton's Results. Journal of Political Economy, 97(3), 740-744. https://doi.org/10.1086/261625

Petrecolla, D. (2006). Costa Rica. Agrocadena del arroz. Estudio Sectorial de Competencia. (Project Report prepared for The World Bank. Manuscript). Recuperado de https://www.coprocom.go.cr/ publicaciones/informes/InformeFinalArroz.pdf

Potter, S. M. (2000). Nonlinear impulse response functions. Journal of Economic Dynamics and Control, 24(10), 1425-1446. https://doi.org/10.1016/S0165-1889(99)00013-5 
ANEXO

CUADRO 1

PRUEBAS DE CAUSALIDAD DE GRANGER

\begin{tabular}{|c|c|c|c|}
\hline \multirow[b]{2}{*}{ Materia prima } & \multicolumn{3}{|c|}{$\begin{array}{l}\text { P-Value asociado a Ho: el IPC no es causado en el } \\
\text { sentido de Granger por: }\end{array}$} \\
\hline & $\begin{array}{c}\text { Precio de } \\
\text { Materia Prima }\end{array}$ & $\mathrm{TC}$ & $\begin{array}{l}\text { Precio de Materia } \\
\text { Prima y TC }\end{array}$ \\
\hline Energy & 0,0257 & 0,0387 & 0,0064 \\
\hline No energía & 0,0028 & 0,0066 & 0,0025 \\
\hline Bienes agrícolas & 0,0422 & 0,0126 & 0,0100 \\
\hline Bebidas & 0,9754 & 0,0443 & 0,2001 \\
\hline Alimentos & 0,0017 & 0,0041 & 0,0007 \\
\hline Aceites comestibles & 0,0000 & 0,0483 & 0,0001 \\
\hline Granos & 0,0174 & 0,0170 & 0,0059 \\
\hline Otros alimentos & 0,7547 & 0,0114 & 0,0393 \\
\hline Materiales básicos & 0,8065 & 0,0112 & 0,0401 \\
\hline Madera & 0,1707 & 0,0133 & 0,0158 \\
\hline Otros materiales básicos & 0,1884 & 0,0137 & 0,0388 \\
\hline Fertilizantes & 0,0010 & 0,0012 & 0,0004 \\
\hline Minerales metálicos & 0,1067 & 0,0063 & 0,0109 \\
\hline Metales básicos & 0,1429 & 0,0076 & 0,0137 \\
\hline Metales preciosos & 0,0156 & 0,0560 & 0,0201 \\
\hline
\end{tabular}

Fuente: Elaboración propia

CUADRO 2

PRUEBAS DE SIMETRÍA EN PARÁMETROS*

\begin{tabular}{lrrrr}
\hline & \multicolumn{2}{c}{ Versión Mork(1989) } & \multicolumn{2}{c}{ Versiónn Mork(1989) modificada } \\
\hline Materia prima & $\begin{array}{c}\text { Estadístico } \\
\mathrm{Ji}^{\wedge} 2\end{array}$ & $\begin{array}{c}\text { P-Value } \\
\text { asociado }\end{array}$ & Estadístico Ji^2 & $\begin{array}{c}\text { P-Value } \\
\text { asociado }\end{array}$ \\
\hline Energy & 3,00 & 0,39 & 4,61 & 0,20 \\
No energía & 2,02 & 0,92 & 1,93 & 0,93
\end{tabular}

Fuente: Elaboración propia 
CUADRO 3

PRUEBAS DE SIMETRÍA SOBRE LOS IMPULSO RESPUESTA

\begin{tabular}{|c|c|c|c|c|}
\hline \multirow[t]{2}{*}{ Calidad de la educación } & \multicolumn{2}{|c|}{ Energía } & \multicolumn{2}{|c|}{ No energía } \\
\hline & 1 desv. Std. & 2 desv. Std. & 1 desv. Std. & 2 desv. Std. \\
\hline 0 & 0,010 & 0,009 & 0,890 & 0,896 \\
\hline 1 & 0,023 & 0,019 & 0,854 & 0,868 \\
\hline 2 & 0,051 & 0,044 & 0,955 & 0,962 \\
\hline 3 & 0,096 & 0,081 & 0,930 & 0,943 \\
\hline 4 & 0,162 & 0,140 & 0,775 & 0,810 \\
\hline 5 & 0,241 & 0,210 & 0,862 & 0,888 \\
\hline 6 & 0,295 & 0,259 & 0,867 & 0,893 \\
\hline 7 & 0,380 & 0,340 & 0,921 & 0,939 \\
\hline 8 & 0,423 & 0,405 & 0,955 & 0,967 \\
\hline 9 & 0,516 & 0,498 & 0,976 & 0,983 \\
\hline 10 & 0,606 & 0,588 & 0,987 & 0,992 \\
\hline 11 & 0,687 & 0,670 & 0,994 & 0,996 \\
\hline 12 & 0,753 & 0,740 & 0,997 & 0,998 \\
\hline 13 & 0,813 & 0,800 & 0,999 & 0,999 \\
\hline 14 & 0,861 & 0,852 & 0,999 & 1,000 \\
\hline 15 & 0,898 & 0,890 & 1,000 & 1,000 \\
\hline 16 & 0,927 & 0,921 & 1,000 & 1,000 \\
\hline 17 & 0,949 & 0,945 & 1,000 & 1,000 \\
\hline 18 & 0,962 & 0,960 & 1,000 & 1,000 \\
\hline 19 & 0,975 & 0,973 & 1,000 & 1,000 \\
\hline 20 & 0,984 & 0,982 & 1,000 & 1,000 \\
\hline 21 & 0,989 & 0,989 & 1,000 & 1,000 \\
\hline 22 & 0,993 & 0,992 & 1,000 & 1,000 \\
\hline 23 & 0,995 & 0,995 & 1,000 & 1,000 \\
\hline
\end{tabular}

Fuente: Elaboración propia

CUADRO 4

PRUEBAS DE SIMETRÍA EN PARÁMETROS CON SERIES REESCALADAS POR VOLATILIDAD*.

\begin{tabular}{lrrrr}
\hline & \multicolumn{2}{c}{ Versión Mork(1989) } & Versiónn Mork(1989) modificada \\
\hline Materia prima & $\begin{array}{c}\text { Estadístico } \\
\mathrm{Ji}^{\wedge} 2\end{array}$ & $\begin{array}{c}\text { P-Value } \\
\text { asociado }\end{array}$ & Estadístico Ji^2 & $\begin{array}{c}\text { P-Value } \\
\text { asociado }\end{array}$ \\
\hline Energy & 2,30 & 0,51 & 2,22 & 0,53 \\
No energía & 1,34 & 0,97 & 1,64 & 0,95
\end{tabular}

Fuente: Elaboración propia 
CUADRO 5

PRUEBAS DE SIMETRÍA SOBRE FUNCIONES IR CON PRECIOS REESCALADOS POR VOLATILIDAD

\begin{tabular}{|c|c|c|c|c|}
\hline \multirow{3}{*}{ Calidad de la educación } & \multicolumn{4}{|c|}{ Valores P, Ho: Impulso respuesta simétrico } \\
\hline & \multicolumn{2}{|c|}{ Energía } & \multicolumn{2}{|c|}{ No energía } \\
\hline & 1 desv. Std. & 2 desv. Std. & 1 desv. Std. & 2 desv. Std. \\
\hline 0 & 0,034 & 0,040 & 0,440 & 0,464 \\
\hline 1 & 0,070 & 0,081 & 0,705 & 0,730 \\
\hline 2 & 0,149 & 0,169 & 0,782 & 0,809 \\
\hline 3 & 0,210 & 0,236 & 0,794 & 0,822 \\
\hline 4 & 0,320 & 0,354 & 0,835 & 0,860 \\
\hline 5 & 0,439 & 0,477 & 0,860 & 0,881 \\
\hline 6 & 0,542 & 0,584 & 0,911 & 0,927 \\
\hline 7 & 0,637 & 0,679 & 0,951 & 0,961 \\
\hline 8 & 0,730 & 0,768 & 0,974 & 0,981 \\
\hline 9 & 0,805 & 0,838 & 0,987 & 0,991 \\
\hline 10 & 0,862 & 0,889 & 0,994 & 0,996 \\
\hline 11 & 0,906 & 0,927 & 0,997 & 0,998 \\
\hline 12 & 0,938 & 0,954 & 0,999 & 0,999 \\
\hline 13 & 0,961 & 0,971 & 0,999 & 1,000 \\
\hline 14 & 0,974 & 0,982 & 1,000 & 1,000 \\
\hline 15 & 0,983 & 0,988 & 1,000 & 1,000 \\
\hline 16 & 0,990 & 0,993 & 1,000 & 1,000 \\
\hline 17 & 0,994 & 0,996 & 1,000 & 1,000 \\
\hline 18 & 0,997 & 0,998 & 1,000 & 1,000 \\
\hline 19 & 0,998 & 0,999 & 1,000 & 1,000 \\
\hline 20 & 0,999 & 0,999 & 1,000 & 1,000 \\
\hline 21 & 0,999 & 1,000 & 1,000 & 1,000 \\
\hline 22 & 1,000 & 1,000 & 1,000 & 1,000 \\
\hline 23 & 1,000 & 1,000 & 1,000 & 1,000 \\
\hline
\end{tabular}

Fuente: Elaboración propia 
CUADRO 6

VALORES P PARA LA PRUEBA DE SIMETRÍA EN LOS IMPULSOS RESPUESTA*

\begin{tabular}{|c|c|c|c|c|c|c|c|c|c|c|c|c|}
\hline \multirow[b]{2}{*}{$\mathrm{h}$} & \multicolumn{2}{|c|}{$\begin{array}{c}\text { Harina y } \\
\text { subproductos } \\
\text { de harina (IPP- } \\
\text { MAN) }\end{array}$} & \multicolumn{2}{|c|}{$\begin{array}{c}\text { Macarrones } \\
\text { y fideos } \\
\text { (IPP-MAN) }\end{array}$} & \multicolumn{2}{|c|}{$\begin{array}{c}\text { Pan cuadrado } \\
\text { (IPC) }\end{array}$} & \multicolumn{2}{|c|}{ Pastas (IPC) } & \multicolumn{2}{|c|}{$\begin{array}{l}\text { Harina de trigo } \\
\text { (IPC) }\end{array}$} & \multicolumn{2}{|c|}{$\begin{array}{c}\text { Alimento para } \\
\text { ganado (IPP- } \\
\text { MAN) }\end{array}$} \\
\hline & 1 d.e & 2 d.e & 1 d.e & 2 d.e & 1 d.e & 2 d.e & 1 d.e & 2 d.e & 1 d.e & 2 d.e & 1 d.e & 2 d.e \\
\hline 0 & 0,262 & 0,326 & 0,003 & 0,003 & 0,062 & 0,088 & 0,640 & 0,657 & 0,293 & 0,341 & 0,217 & 0,240 \\
\hline 1 & 0,209 & 0,300 & 0,001 & 0,001 & 0,002 & 0,003 & 0,033 & 0,040 & 0,553 & 0,609 & 0,048 & 0,064 \\
\hline 2 & 0,350 & 0,473 & 0,002 & 0,002 & 0,005 & 0,009 & 0,074 & 0,088 & 0,077 & 0,114 & 0,101 & 0,131 \\
\hline 3 & 0,000 & 0,001 & 0,006 & 0,005 & 0,011 & 0,019 & 0,118 & 0,139 & 0,038 & 0,061 & 0,122 & 0,158 \\
\hline 4 & 0,000 & 0,001 & 0,012 & 0,010 & 0,022 & 0,036 & 0,194 & 0,223 & 0,07 & 0,106 & 0,194 & 0,246 \\
\hline 5 & 0,001 & 0,003 & 0,024 & 0,020 & 0,039 & 0,063 & 0,285 & 0,323 & 0,114 & 0,165 & 0,287 & 0,350 \\
\hline 6 & 0,000 & 0,002 & 0,041 & 0,034 & 0,011 & 0,017 & 0,372 & 0,415 & 0,174 & 0,241 & 0,101 & 0,134 \\
\hline 7 & 0,001 & 0,004 & 0,061 & 0,051 & 0,019 & 0,03 & 0,475 & 0,522 & 0,149 & 0,204 & 0,036 & 0,051 \\
\hline 8 & 0,002 & 0,007 & 0,093 & 0,079 & 0,032 & 0,048 & 0,561 & 0,616 & 0,207 & 0,273 & 0,046 & 0,065 \\
\hline 9 & 0,003 & 0,011 & 0,133 & 0,115 & 0,031 & 0,046 & 0,653 & 0,705 & 0,276 & 0,354 & 0,071 & 0,097 \\
\hline 10 & 0,005 & 0,017 & 0,177 & 0,158 & 0,046 & 0,065 & 0,723 & 0,773 & 0,081 & 0,098 & 0,102 & 0,136 \\
\hline 11 & 0,008 & 0,027 & 0,233 & 0,209 & 0,061 & 0,09 & 0,794 & 0,835 & 0,112 & 0,135 & 0,141 & 0,184 \\
\hline 12 & 0,003 & 0,011 & 0,279 & 0,250 & 0,051 & 0,068 & 0,507 & 0,562 & 0,134 & 0,159 & 0,185 & 0,236 \\
\hline 13 & 0,005 & 0,018 & 0,345 & 0,314 & 0,072 & 0,095 & 0,585 & 0,639 & 0,178 & 0,208 & 0,235 & 0,297 \\
\hline 14 & 0,008 & 0,026 & 0,416 & 0,382 & 0,1 & 0,128 & 0,659 & 0,709 & 0,225 & 0,263 & 0,295 & 0,362 \\
\hline 15 & 0,012 & 0,038 & 0,488 & 0,452 & 0,131 & 0,167 & 0,726 & 0,771 & 0,242 & 0,289 & 0,357 & 0,428 \\
\hline 16 & 0,017 & 0,053 & 0,557 & 0,523 & 0,169 & 0,212 & 0,784 & 0,824 & 0,299 & 0,35 & 0,424 & 0,498 \\
\hline 17 & 0,024 & 0,072 & 0,621 & 0,590 & 0,213 & 0,263 & 0,833 & 0,867 & 0,358 & 0,413 & 0,492 & 0,567 \\
\hline 18 & 0,034 & 0,094 & 0,684 & 0,653 & 0,251 & 0,307 & 0,874 & 0,902 & 0,421 & 0,478 & 0,558 & 0,633 \\
\hline 19 & 0,046 & 0,122 & 0,739 & 0,709 & 0,3 & 0,361 & 0,903 & 0,926 & 0,476 & 0,527 & 0,622 & 0,691 \\
\hline 20 & 0,063 & 0,155 & 0,790 & 0,761 & 0,356 & 0,42 & 0,929 & 0,947 & 0,538 & 0,59 & 0,680 & 0,743 \\
\hline 21 & 0,082 & 0,192 & 0,831 & 0,807 & 0,412 & 0,478 & 0,949 & 0,963 & 0,591 & 0,647 & 0,730 & 0,789 \\
\hline 22 & 0,100 & 0,226 & 0,868 & 0,847 & 0,47 & 0,538 & 0,963 & 0,973 & 0,643 & 0,699 & 0,778 & 0,831 \\
\hline 23 & 0,126 & 0,272 & 0,894 & 0,877 & 0,505 & 0,583 & 0,973 & 0,982 & 0,694 & 0,75 & 0,821 & 0,868 \\
\hline 24 & 0,126 & 0,272 & 0,894 & 0,877 & 0,505 & 0,583 & 0,973 & 0,982 & 0,694 & 0,75 & 0,821 & 0,868 \\
\hline 25 & 0,157 & 0,320 & 0,920 & 0,905 & 0,562 & 0,639 & 0,981 & 0,987 & 0,744 & 0,795 & 0,858 & 0,898 \\
\hline 26 & 0,189 & 0,371 & 0,939 & 0,928 & 0,615 & 0,689 & 0,987 & 0,992 & 0,78 & 0,825 & 0,887 & 0,919 \\
\hline 27 & 0,227 & 0,424 & 0,955 & 0,947 & 0,653 & 0,725 & 0,991 & 0,995 & 0,82 & 0,86 & 0,912 & 0,938 \\
\hline 28 & 0,269 & 0,477 & 0,966 & 0,960 & 0,702 & 0,77 & 0,994 & 0,997 & 0,843 & 0,88 & 0,931 & 0,952 \\
\hline 29 & 0,312 & 0,530 & 0,974 & 0,970 & 0,748 & 0,81 & 0,996 & 0,998 & 0,872 & 0,903 & 0,948 & 0,964 \\
\hline 30 & 0,358 & 0,581 & 0,981 & 0,979 & 0,788 & 0,845 & 0,998 & 0,999 & 0,899 & 0,925 & 0,961 & 0,974 \\
\hline 31 & 0,379 & 0,594 & 0,987 & 0,985 & 0,818 & 0,868 & 0,999 & 0,999 & 0,921 & 0,943 & 0,966 & 0,977 \\
\hline 32 & 0,427 & 0,642 & 0,991 & 0,989 & 0,851 & 0,894 & 0,999 & 0,999 & 0,939 & 0,957 & 0,975 & 0,983 \\
\hline 33 & 0,473 & 0,688 & 0,994 & 0,993 & 0,87 & 0,912 & 0,999 & 1 & 0,954 & 0,967 & 0,981 & 0,988 \\
\hline 34 & 0,522 & 0,731 & 0,996 & 0,995 & 0,893 & 0,93 & 1 & 1 & 0,965 & 0,976 & 0,986 & 0,991 \\
\hline 35 & 0,570 & 0,771 & 0,997 & 0,996 & 0,915 & 0,946 & 1 & 1 & 0,974 & 0,983 & 0,990 & 0,994 \\
\hline 36 & 0,615 & 0,803 & 0,998 & 0,998 & 0,932 & 0,958 & 1 & 1 & 0,981 & 0,987 & 0,993 & 0,996 \\
\hline
\end{tabular}




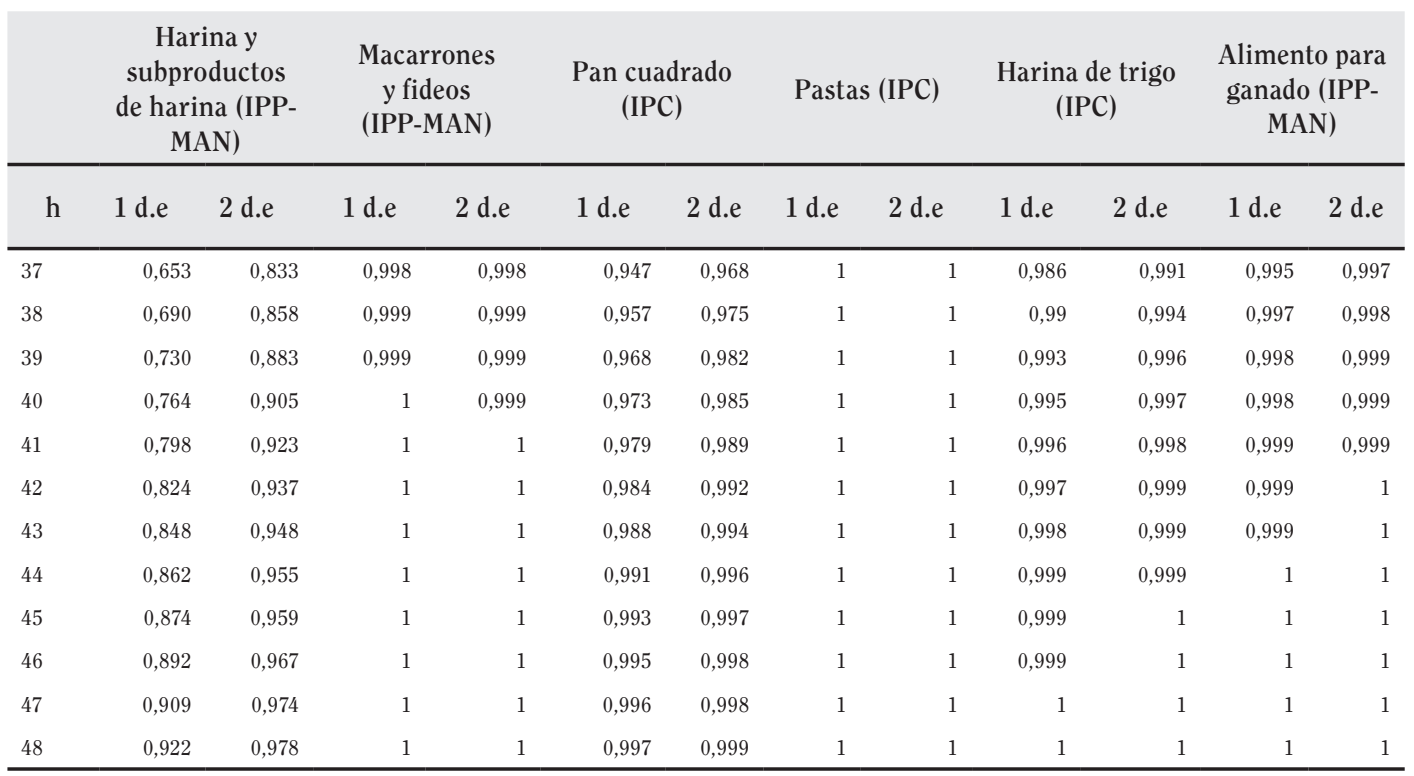

\begin{tabular}{|c|c|c|c|c|c|c|c|c|c|c|c|c|}
\hline \multicolumn{3}{|c|}{$\begin{array}{c}\text { Polloy } \\
\text { productos } \\
\text { de pollo } \\
\text { (IPP-MAN) }\end{array}$} & \multicolumn{2}{|c|}{$\begin{array}{c}\text { Pollo } \\
\text { (IPP-MAN) }\end{array}$} & \multicolumn{2}{|c|}{$\begin{array}{l}\text { Pechuga de } \\
\text { pollo (IPC) }\end{array}$} & \multicolumn{2}{|c|}{$\begin{array}{l}\text { Muslo de pollo } \\
\text { (IPC) }\end{array}$} & \multicolumn{2}{|c|}{$\begin{array}{l}\text { Pollo entero } \\
\text { (IPC) }\end{array}$} & \multicolumn{2}{|c|}{$\begin{array}{l}\text { Alas de pollo } \\
\text { (IPC) }\end{array}$} \\
\hline $\mathrm{h}$ & 1 d.e & 2 d.e & 1 d.e & 2 d.e & 1 d.e & 2 d.e & 1 d.e & 2 d.e & 1 d.e & 2 d.e & 1 d.e & 2 d.e \\
\hline 1 & 0,230 & 0,248 & 0,311 & 0,331 & 0,036 & 0,037 & 0,616 & 0,635 & 0,009 & 0,010 & 0,047 & 0,045 \\
\hline 2 & 0,099 & 0,112 & 0,034 & 0,045 & 0,014 & 0,015 & 0,025 & 0,031 & 0,003 & 0,002 & 0,002 & 0,002 \\
\hline 3 & 0,006 & 0,006 & 0,000 & 0,001 & 0,036 & 0,039 & 0,046 & 0,056 & 0,007 & 0,006 & 0,005 & 0,004 \\
\hline 4 & 0,014 & 0,015 & 0,001 & 0,002 & 0,074 & 0,078 & 0,088 & 0,105 & 0,016 & 0,014 & 0,010 & 0,009 \\
\hline 5 & 0,018 & 0,018 & 0,001 & 0,002 & 0,127 & 0,133 & 0,151 & 0,175 & 0,032 & 0,028 & 0,018 & 0,017 \\
\hline 6 & 0,033 & 0,034 & 0,003 & 0,005 & 0,192 & 0,204 & 0,230 & 0,263 & 0,054 & 0,049 & 0,032 & 0,032 \\
\hline 7 & 0,056 & 0,057 & 0,006 & 0,010 & 0,276 & 0,290 & 0,321 & 0,361 & 0,086 & 0,080 & 0,055 & 0,055 \\
\hline 8 & 0,076 & 0,084 & 0,010 & 0,016 & 0,348 & 0,368 & 0,419 & 0,464 & 0,130 & 0,119 & 0,083 & 0,083 \\
\hline 9 & 0,114 & 0,125 & 0,017 & 0,028 & 0,435 & 0,457 & 0,519 & 0,565 & 0,186 & 0,172 & 0,124 & 0,124 \\
\hline 10 & 0,162 & 0,176 & 0,027 & 0,042 & 0,530 & 0,552 & 0,603 & 0,651 & 0,247 & 0,234 & 0,175 & 0,175 \\
\hline 11 & 0,217 & 0,234 & 0,042 & 0,063 & 0,600 & 0,619 & 0,687 & 0,732 & 0,318 & 0,302 & 0,235 & 0,234 \\
\hline 12 & 0,281 & 0,302 & 0,060 & 0,090 & 0,664 & 0,684 & 0,761 & 0,801 & 0,393 & 0,383 & 0,306 & 0,303 \\
\hline 13 & 0,351 & 0,373 & 0,086 & 0,124 & 0,744 & 0,756 & 0,819 & 0,853 & 0,467 & 0,477 & 0,387 & 0,374 \\
\hline 14 & 0,424 & 0,449 & 0,117 & 0,164 & 0,862 & 0,817 & 0,862 & 0,891 & 0,546 & 0,556 & 0,464 & 0,450 \\
\hline 15 & 0,499 & 0,525 & 0,154 & 0,212 & 0,787 & 0,866 & 0,896 & 0,920 & 0,641 & 0,631 & 0,540 & 0,526 \\
\hline 16 & 0,568 & 0,596 & 0,195 & 0,263 & 0,839 & 0,908 & 0,928 & 0,946 & 0,794 & 0,867 & 0,613 & 0,599 \\
\hline 17 & 0,630 & 0,654 & 0,245 & 0,322 & 0,881 & 0,936 & 0,951 & 0,964 & 0,843 & 0,940 & 0,680 & 0,667 \\
\hline 18 & 0,694 & 0,716 & 0,300 & 0,384 & 0,914 & 0,957 & 0,968 & 0,977 & 0,883 & 0,960 & 0,741 & 0,729 \\
\hline
\end{tabular}




\begin{tabular}{|c|c|c|c|c|c|c|c|c|c|c|c|c|}
\hline \multicolumn{3}{|c|}{$\begin{array}{c}\text { Pollo y } \\
\text { productos } \\
\text { de pollo } \\
\text { (IPP-MAN) }\end{array}$} & \multicolumn{2}{|c|}{$\begin{array}{c}\text { Pollo } \\
\text { (IPP-MAN) }\end{array}$} & \multicolumn{2}{|c|}{$\begin{array}{l}\text { Pechuga de } \\
\text { pollo (IPC) }\end{array}$} & \multicolumn{2}{|c|}{$\begin{array}{l}\text { Muslo de pollo } \\
\text { (IPC) }\end{array}$} & \multicolumn{2}{|c|}{$\begin{array}{l}\text { Pollo entero } \\
\text { (IPC) }\end{array}$} & \multicolumn{2}{|c|}{$\begin{array}{l}\text { Alas de pollo } \\
\text { (IPC) }\end{array}$} \\
\hline $\mathrm{h}$ & 1 d.e & 2 d.e & 1 d.e & 2 d.e & 1 d.e & 2 d.e & 1 d.e & 2 d.e & 1 d.e & 2 d.e & 1 d.e & 2 d.e \\
\hline 19 & 0,752 & 0,772 & 0,360 & 0,448 & 0,940 & 0,971 & 0,979 & 0,985 & 0,915 & 0,973 & 0,795 & 0,784 \\
\hline 20 & 0,799 & 0,818 & 0,418 & 0,507 & 0,958 & 0,981 & 0,987 & 0,991 & 0,939 & 0,983 & 0,840 & 0,830 \\
\hline 21 & 0,842 & 0,859 & 0,480 & 0,570 & 0,972 & 0,991 & 0,992 & 0,994 & 0,958 & 0,989 & 0,878 & 0,870 \\
\hline 22 & 0,878 & 0,892 & 0,542 & 0,630 & 0,981 & 0,995 & 0,995 & 0,997 & 0,971 & 0,993 & 0,908 & 0,901 \\
\hline 23 & 0,907 & 0,919 & 0,602 & 0,687 & 0,988 & 0,997 & 0,997 & 0,998 & 0,980 & 0,996 & 0,932 & 0,927 \\
\hline 24 & 0,931 & 0,940 & 0,637 & 0,724 & 0,992 & 0,998 & 0,998 & 0,999 & 0,987 & 0,998 & 0,951 & 0,947 \\
\hline 24 & 0,126 & 0,272 & 0,894 & 0,877 & 0,505 & 0,583 & 0,973 & 0,982 & 0,694 & 0,75 & 0,821 & 0,868 \\
\hline 25 & 0,949 & 0,956 & 0,690 & 0,771 & 0,995 & 0,999 & 0,999 & 0,999 & 0,992 & 0,999 & 0,965 & 0,962 \\
\hline 26 & 0,961 & 0,965 & 0,737 & 0,808 & 0,984 & 0,999 & 0,999 & 1,000 & 0,995 & 0,999 & 0,975 & 0,973 \\
\hline 27 & 0,972 & 0,975 & 0,782 & 0,846 & 0,999 & 1,000 & 1,000 & 1,000 & 0,997 & 1,000 & 0,983 & 0,945 \\
\hline 28 & 0,980 & 0,982 & 0,821 & 0,877 & 0,999 & 1,000 & 1,000 & 1,000 & 0,998 & 1,000 & 0,988 & 0,960 \\
\hline 29 & 0,986 & 0,988 & 0,855 & 0,903 & 1,000 & 1,000 & 1,000 & 1,000 & 0,999 & 1,000 & 0,992 & 0,971 \\
\hline 30 & 0,990 & 0,992 & 0,884 & 0,925 & 1,000 & 1,000 & 1,000 & 1,000 & 0,999 & 1,000 & 0,995 & 0,979 \\
\hline 31 & 0,993 & 0,994 & 0,907 & 0,942 & 1,000 & 1,000 & 1,000 & 1,000 & 1,000 & 1,000 & 0,997 & 0,985 \\
\hline 32 & 0,996 & 0,996 & 0,926 & 0,955 & 1,000 & 1,000 & 1,000 & 1,000 & 1,000 & 1,000 & 0,998 & 0,990 \\
\hline 33 & 0,997 & 0,998 & 0,943 & 0,966 & 1,000 & 1,000 & 1,000 & 1,000 & 1,000 & 1,000 & 0,999 & 0,993 \\
\hline 34 & 0,998 & 0,998 & 0,954 & 0,973 & 1,000 & 1,000 & 1,000 & 1,000 & 1,000 & 1,000 & 0,999 & 0,995 \\
\hline 35 & 0,999 & 0,999 & 0,963 & 0,979 & 1,000 & 1,000 & 1,000 & 1,000 & 1,000 & 1,000 & 0,999 & 0,997 \\
\hline 36 & 0,999 & 0,999 & 0,972 & 0,985 & 1,000 & 1,000 & 1,000 & 1,000 & 1,000 & 1,000 & 1,000 & 0,998 \\
\hline 37 & 0,999 & 1,000 & 0,979 & 0,989 & 1,000 & 1,000 & 1,000 & 1,000 & 1,000 & 1,000 & 1,000 & 0,999 \\
\hline 38 & 1,000 & 1,000 & 0,984 & 0,992 & 1,000 & 1,000 & 1,000 & 1,000 & 1,000 & 1,000 & 1,000 & 0,999 \\
\hline 39 & 1,000 & 1,000 & 0,987 & 0,994 & 1,000 & 1,000 & 1,000 & 1,000 & 1,000 & 1,000 & 1,000 & 0,999 \\
\hline 40 & 1,000 & 1,000 & 0,991 & 0,996 & 1,000 & 1,000 & 1,000 & 1,000 & 1,000 & 1,000 & 1,000 & 1,000 \\
\hline 41 & 1,000 & 1,000 & 0,993 & 0,997 & 1,000 & 1,000 & 1,000 & 1,000 & 1,000 & 1,000 & 1,000 & 1,000 \\
\hline 42 & 1,000 & 1,000 & 0,995 & 0,998 & 1,000 & 1,000 & 1,000 & 1,000 & 1,000 & 1,000 & 1,000 & 1,000 \\
\hline 43 & 1,000 & 1,000 & 0,997 & 0,999 & 1,000 & 1,000 & 1,000 & 1,000 & 1,000 & 1,000 & 1,000 & 1,000 \\
\hline 44 & 1,000 & 1,000 & 0,998 & 0,999 & 1,000 & 1,000 & 1,000 & 1,000 & 1,000 & 1,000 & 1,000 & 1,000 \\
\hline 45 & 1,000 & 1,000 & 0,998 & 0,999 & 1,000 & 1,000 & 1,000 & 1,000 & 1,000 & 1,000 & 1,000 & 1,000 \\
\hline 46 & 1,000 & 1,000 & 0,999 & 1,000 & 1,000 & 1,000 & 1,000 & 1,000 & 1,000 & 1,000 & 1,000 & 1,000 \\
\hline 47 & 1,000 & 1,000 & 0,999 & 1,000 & 1,000 & 1,000 & 1,000 & 1,000 & 1,000 & 1,000 & 1,000 & 1,000 \\
\hline 48 & 1,000 & 1,000 & 0,999 & 1,000 & 1,000 & 1,000 & 1,000 & 1,000 & 1,000 & 1,000 & 1,000 & 1,000 \\
\hline
\end{tabular}

** Valores en negrita indican significancia al 5\%

Fuente: Elaboración propia 


\section{CUADRO 7 \\ TRASPASO ACUMULADO}

\begin{tabular}{|c|c|c|c|c|c|c|c|c|}
\hline & \multicolumn{2}{|c|}{$\begin{array}{c}\text { Harina y } \\
\text { subproductos de } \\
\text { harina (IPP-MAN) }\end{array}$} & \multicolumn{2}{|c|}{$\begin{array}{l}\text { Macarrones y fideos } \\
\text { (IPP-MAN) }\end{array}$} & \multicolumn{2}{|c|}{$\begin{array}{l}\text { Pan cuadrado } \\
\text { (IPC) }\end{array}$} & \multicolumn{2}{|c|}{ Pastas (IPC) } \\
\hline \multicolumn{9}{|c|}{ Choque positivo } \\
\hline $\mathrm{h}$ & 1 d.e & 2 d.e & 1 d.e & 2 d.e & 1 d.e & 2 d.e & 1 d.e & 2 d.e \\
\hline 12 & 4,57 & 5,03 & 2,24 & 2,08 & 0,79 & 0,85 & 0,87 & 1,27 \\
\hline 24 & 3,91 & 4,14 & 1,99 & 1,91 & 0,91 & 0,94 & 0,80 & 1,43 \\
\hline 36 & 4,01 & 4,27 & 1,95 & 1,86 & 0,91 & 0,94 & 0,60 & 1,15 \\
\hline 48 & 4,00 & 4,27 & 1,97 & 1,88 & 0,95 & 0,98 & 0,61 & 1,21 \\
\hline \multicolumn{9}{|c|}{ Choque negativo } \\
\hline $\mathrm{H}$ & 1 d.e & 2 d.e & 1 d.e & 2 d.e & 1 d.e & 2 d.e & 1 d.e & 2 d.e \\
\hline 12 & 4,19 & 3,70 & 2,37 & 2,55 & 0,72 & 0,64 & 0,56 & 0,18 \\
\hline 24 & 3,74 & 3,49 & 2,06 & 2,15 & 0,88 & 0,83 & 0,31 & $-0,28$ \\
\hline 36 & 3,80 & 3,52 & 2,02 & 2,11 & 0,88 & 0,83 & 0,19 & $-0,33$ \\
\hline \multirow[t]{2}{*}{48} & 3,81 & 3,54 & 2,04 & 2,13 & 0,92 & 0,88 & 0,16 & $-0,40$ \\
\hline & \multicolumn{2}{|c|}{$\begin{array}{l}\text { Harina de trigo } \\
\text { (IPC) }\end{array}$} & \multicolumn{2}{|c|}{$\begin{array}{c}\text { Alimento para } \\
\text { ganado (IPP-MAN) }\end{array}$} & \multicolumn{2}{|c|}{$\begin{array}{l}\text { Productos de pollo } \\
\quad \text { (IPP-MAN) }\end{array}$} & \multicolumn{2}{|c|}{ Pollo (IPP-MAN) } \\
\hline \multicolumn{9}{|c|}{ Choque positivo } \\
\hline $\mathrm{h}$ & 1 d.e & 2 d.e & 1 d.e & 2 d.e & 1 d.e & 2 d.e & 1 d.e & 2 d.e \\
\hline $\mathrm{H}$ & 1 d.e & 2 d.e & 1 d.e & 2 d.e & 1 d.e & 2 d.e & 1 d.e & 2 d.e \\
\hline 12 & 3,10 & 3,00 & 1,028 & 1,465 & 0,90 & 1,21 & 0,97 & 1,30 \\
\hline 24 & 2,68 & 2,45 & 0,653 & 1,114 & 0,89 & 1,20 & 0,96 & 1,28 \\
\hline 36 & 2,77 & 2,54 & 0,635 & 1,085 & 0,89 & 1,20 & 0,96 & 1,28 \\
\hline 48 & 2,71 & 2,47 & 0,631 & 1,130 & 0,89 & 1,20 & 0,96 & 1,28 \\
\hline \multicolumn{9}{|c|}{ Choque negativo } \\
\hline $\mathrm{h}$ & 1 d.e & 2 d.e & 1 d.e & 2 d.e & 1 d.e & 2 d.e & 1 d.e & 2 d.e \\
\hline 12 & 3,18 & 3,30 & 0,600 & 0,032 & 0,67 & 0,30 & 0,68 & 0,26 \\
\hline 24 & 2,88 & 3,20 & 0,221 & $-0,374$ & 0,66 & 0,29 & 0,67 & 0,26 \\
\hline 36 & 2,97 & 3,29 & 0,213 & $-0,368$ & 0,66 & 0,29 & 0,67 & 0,26 \\
\hline \multirow[t]{2}{*}{48} & 2,90 & 3,22 & 0,162 & $-0,483$ & 0,66 & 0,30 & 0,67 & 0,26 \\
\hline & \multicolumn{2}{|c|}{$\begin{array}{l}\text { Pechuga de pollo } \\
\text { (IPC) }\end{array}$} & \multicolumn{2}{|c|}{ Muslo de pollo (IPC) } & \multicolumn{2}{|c|}{ Pollo entero (IPC) } & \multicolumn{2}{|c|}{$\begin{array}{l}\text { Alas de pollo } \\
\text { (IPC) }\end{array}$} \\
\hline \multicolumn{9}{|c|}{ Choque positivo } \\
\hline $\mathrm{h}$ & 1 d.e & 2 d.e & 1 d.e & 2 d.e & 1 d.e & 2 d.e & 1 d.e & 2 d.e \\
\hline 12 & 0,16 & 0,37 & 0,84 & 1,11 & 0,17 & 0,48 & 0,35 & 0,70 \\
\hline 24 & 0,15 & 0,36 & 0,87 & 1,14 & 0,17 & 0,48 & 0,34 & 0,70 \\
\hline 36 & 0,14 & 0,35 & 0,88 & 1,15 & 0,16 & 0,48 & 0,33 & 0,69 \\
\hline 48 & 0,13 & 0,34 & 0,89 & 1,16 & 0,16 & 0,47 & 0,33 & 0,69 \\
\hline
\end{tabular}




\begin{tabular}{|c|c|c|c|c|c|c|c|c|}
\hline \multicolumn{9}{|c|}{ Choque negativo } \\
\hline $\mathrm{h}$ & 1 d.e & 2 d.e & 1 d.e & 2 d.e & 1 d.e & 2 d.e & 1 d.e & 2 d.e \\
\hline 12 & $-0,12$ & $-0,37$ & 0,56 & 0,28 & $-0,20$ & $-0,57$ & $-0,03$ & $-0,47$ \\
\hline 24 & $-0,13$ & $-0,38$ & 0,60 & 0,32 & $-0,20$ & $-0,57$ & $-0,04$ & $-0,48$ \\
\hline 36 & $-0,14$ & $-0,39$ & 0,62 & 0,34 & $-0,21$ & $-0,58$ & $-0,05$ & $-0,49$ \\
\hline 48 & $-0,15$ & $-0,41$ & 0,63 & 0,35 & $-0,21$ & $-0,58$ & $-0,05$ & $-0,49$ \\
\hline \multicolumn{3}{|c|}{ Z (IPP-MAN) } & \multicolumn{2}{|c|}{ Arroz (IPC) } & \multicolumn{2}{|c|}{$\begin{array}{c}\text { Hierro y productos } \\
\text { de acero (IPP- } \\
\text { MAN) }\end{array}$} & \multicolumn{2}{|c|}{ Fertilizantes } \\
\hline \multicolumn{9}{|c|}{ Choque positivo } \\
\hline $\mathrm{h}$ & 1 d.e & 2 d.e & 1 d.e & 2 d.e & 1 d.e & 2 d.e & 1 d.e & 2 d.e \\
\hline 12 & 0,03 & $-0,73$ & 1,13 & 0,77 & 1,61 & 0,75 & 7,71 & 8,26 \\
\hline 24 & 0,36 & $-0,22$ & 1,27 & 0,79 & 2,93 & 2,04 & 7,75 & 8,14 \\
\hline 36 & 0,55 & $-0,08$ & 1,53 & 1,03 & 3,06 & 2,10 & 7,58 & 7,95 \\
\hline 48 & 0,71 & 0,06 & 1,72 & 1,21 & 3,16 & 2,19 & 7,76 & 8,14 \\
\hline \multicolumn{9}{|c|}{ Choque negativo } \\
\hline $\mathrm{h}$ & 1 d.e & 2 d.e & 1 d.e & 2 d.e & 1 d.e & 2 d.e & 1 d.e & 2 d.e \\
\hline 12 & 0,83 & $-1,51$ & 1,45 & 0,42 & 2,45 & 3,52 & 7,60 & 6,87 \\
\hline 24 & 1,00 & $-0,88$ & 1,72 & 0,27 & 3,76 & 4,91 & 7,68 & 7,17 \\
\hline 36 & 1,26 & $-0,80$ & 2,00 & 0,48 & 3,96 & 5,19 & 7,51 & 7,01 \\
\hline 48 & 1,45 & $-0,69$ & 2,21 & 0,64 & 4,05 & 5,29 & 7,70 & 7,19 \\
\hline
\end{tabular}

Fuente: Elaboración propia 


\section{GRÁFICO 6 \\ IMPULSO RESPUESTA ACUMULADO DEL IPC ANTE CHOQUES NEGATIVOS SOBRE MATERIAS PRIMAS ENERGÉTICAS}
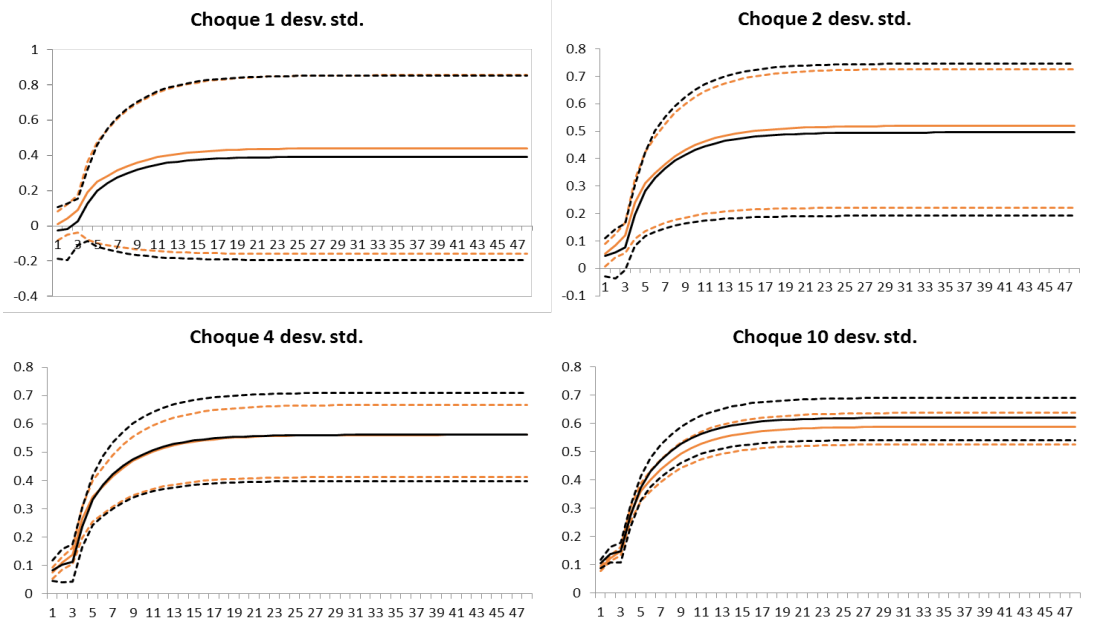

- Con corrección por volatilidad

-..- Intervalo del $90 \%$

Fuente: Elaboración propia

....-Intervalo del $90 \%$

\section{GRÁFICO 7}

IMPULSO RESPUESTA ACUMULADO DEL IPC ANTE CHOQUES POSITIVOS SOBRE MATERIAS PRIMAS NO ENERGÉTICAS.

Choque 1 desv, std.
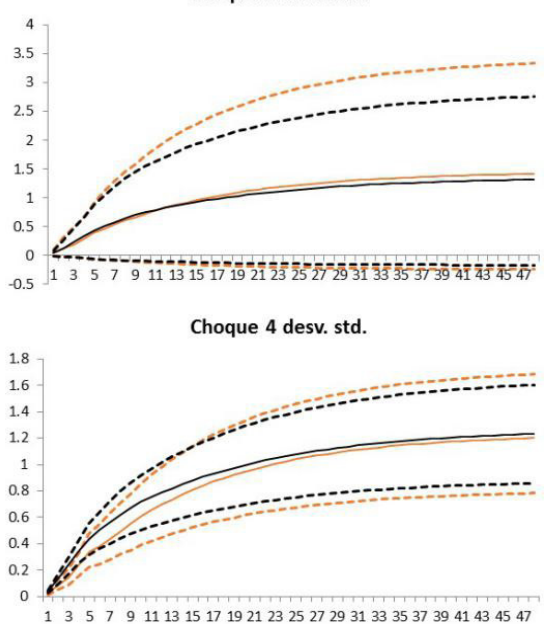

- Con corrección por volatilidad

- Sin corrección por volatilidad

Fuente: Elaboración propia
Choque 2 desv. std.

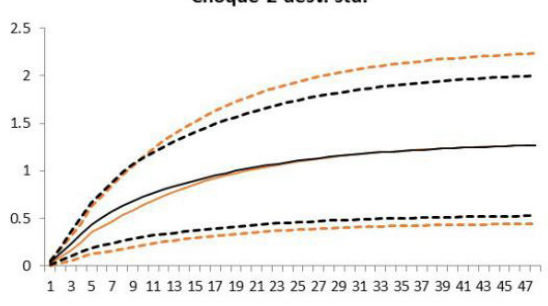

Choque 10 desv. std.

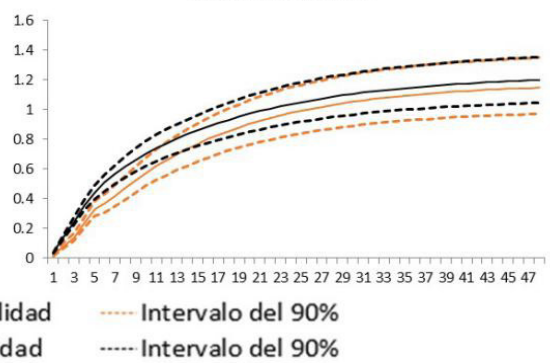




\section{GRÁFICO 8}

IMPULSO RESPUESTA ACUMULADO DEL IPC ANTE CHOQUES NEGATIVOS SOBRE MATERIAS PRIMAS NO ENERGÉTICAS
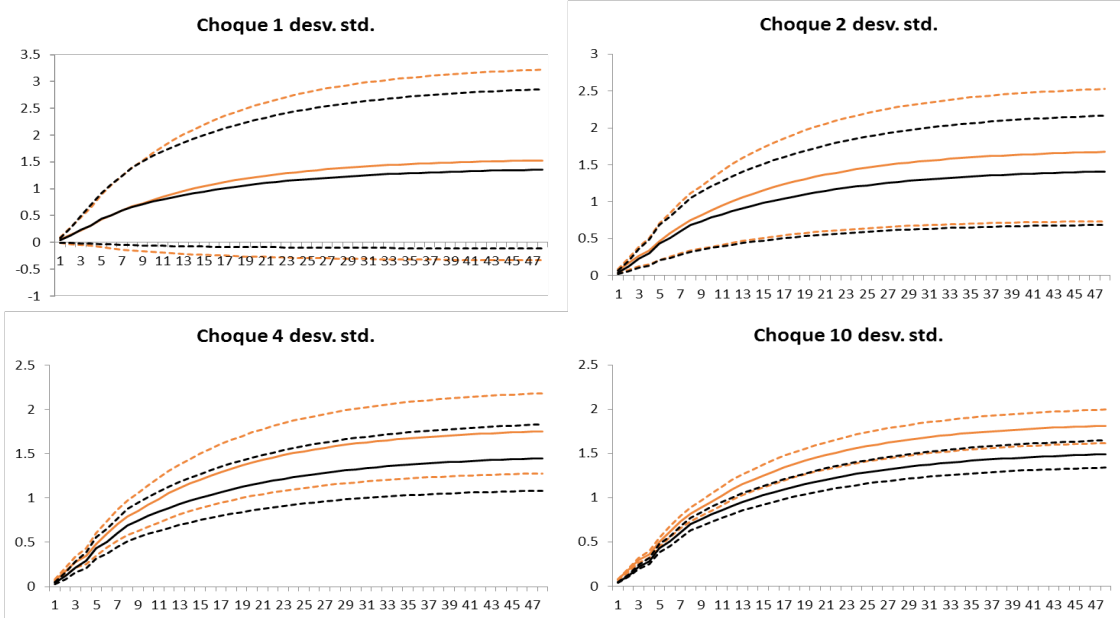

—Con corrección por volatilidad

-..-. Intervalo del $90 \%$

Fuente: Elaboración propia

-...-... Intervalo del $90 \%$

\section{GRÁFICO 9}

IMPULSO RESPUESTA ACUMULADO DE HARINA Y SUBPRODUCTOS DE HARINA (IPP)

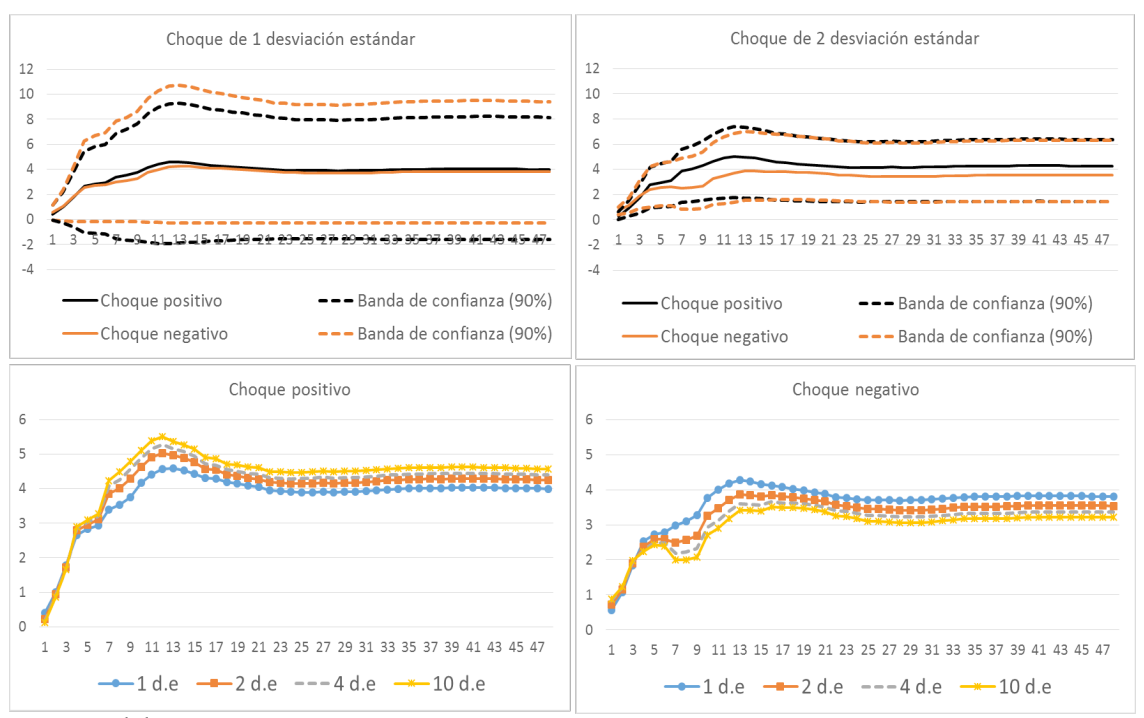

Fuente: Elaboración propia 
GRÁFICO 10

IMPULSO RESPUESTA ACUMULADO DE POLLO Y PRODUCTOS DE POLLO (IPP).
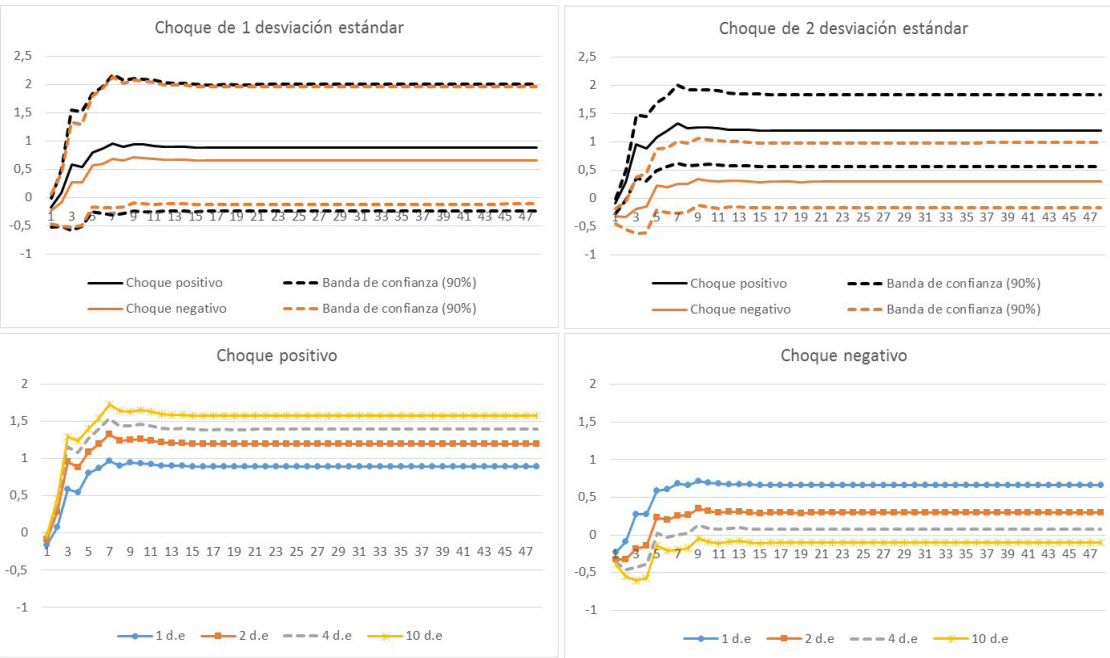

Fuente: Elaboración propia

GRÁFICO 11

IMPULSO RESPUESTA ACUMULADO DE ARROZ (IPP).
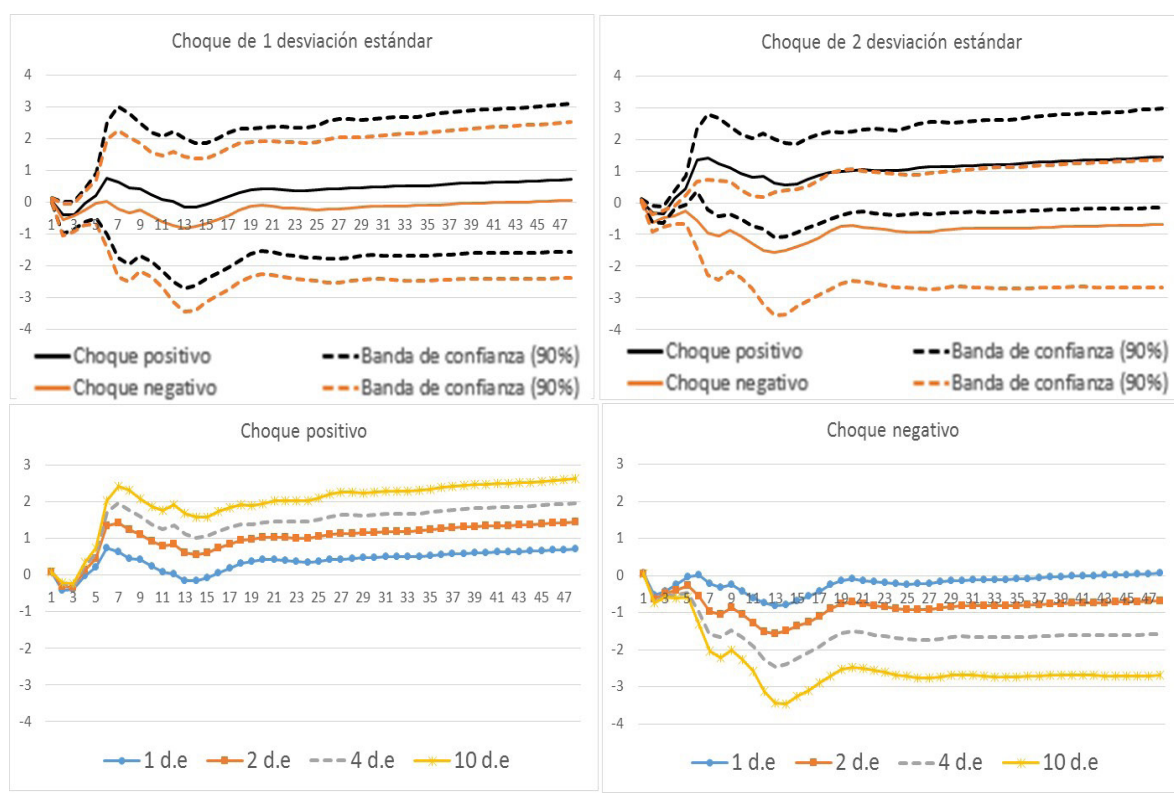

Fuente: Elaboración propia 


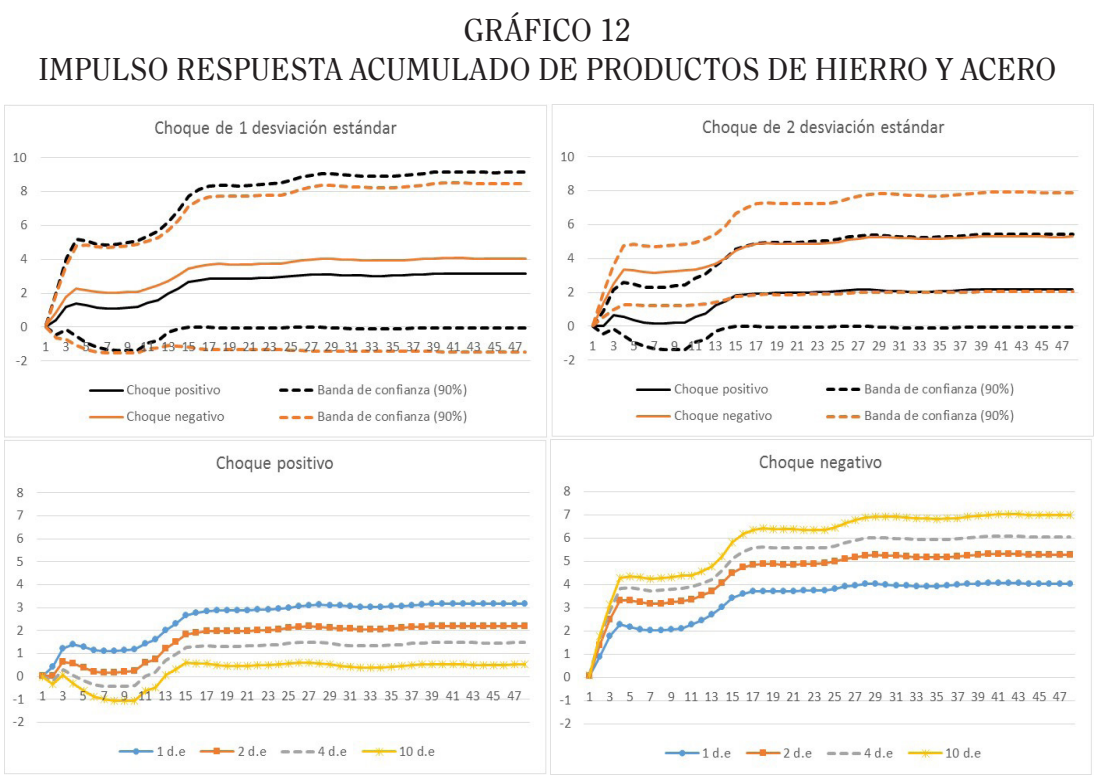

Fuente: Elaboración propia 
